\title{
Implementation of Endomorphisms of the CAR Algebra
}

\author{
Carsten Binnenhei*† \\ Institut für Theoretische Physik der Freien Universität Berlin \\ Arnimallee 14, 14195 Berlin, Germany
}

February 1995

\begin{abstract}
The implementation of non-surjective Bogoliubov transformations in Fock states over CAR algebras is investigated. Such a transformation is implementable by a Hilbert space of isometries if and only if the well-known Shale-Stinespring condition is met. In this case, the dimension of the implementing Hilbert space equals the square root of the Watatani index of the associated inclusion of CAR algebras, and both are determined by the Fredholm index of the corresponding one-particle operator. Explicit expressions for the implementing operators are obtained, and the connected components of the semigroup of implementable transformations are described.
\end{abstract}

\section{Introduction}

The implementation of Bogoliubov automorphisms of the algebra of canonical anticommutation relations (CAR) by unitary operators on Fock space is well-understood. Shale and Stinespring [1] have proven that such an automorphism is implementable in a Fock representation if and only if the corresponding one-particle Bogoliubov operator satisfies a certain Hilbert-Schmidt condition, and several authors (e.g. Friedrichs [2], Berezin [3, Labonté [, Fredenhagen [5], Klaus and Scharf [6], Ruijsenaars [7, 8]) have constructed the implementing unitaries in terms of annihilation and creation operators.

Here we tackle the problem of extending these results to the case of Bogoliubov endomorphisms. As suggested by the work of Doplicher and Roberts [9] on the theory of superselection sectors (see [10] for an overview), the appropriate generalization of 'implementation of automorphisms by unitary operators' is 'implementation of endomorphisms by Hilbert spaces of isometries'. An endomorphism $\varrho$ is implementable in a representation $\pi$ of an arbitrary $\mathrm{C}^{*}$-algebra if and only if $\pi \circ \varrho$ is unitarily equivalent to a multiple of $\pi$, and then the multiplicity equals the dimension of an implementing Hilbert space. For irreducible $\pi$, implementability is tantamount to quasi-equivalence of $\pi$ and $\pi \circ \varrho$.

In the case of Bogoliubov endomorphisms and (irreducible) Fock representations of the CAR algebra, one may apply the criterion for quasi-equivalence of quasi-free states due to Powers and Størmer [11] and Araki [12] to conclude that a Bogoliubov endomorphism is implementable in the above sense if and only if the corresponding Bogoliubov operator fulfills the Shale-Stinespring condition. The dimension of the implementing Hilbert space is then given by the square root of the Watatani index 13] of the associated inclusion of $\mathrm{C}^{*}$-algebras, and this index in turn equals $2^{- \text {ind } V}$ where - ind $V \in 2 \mathbb{N} \cup\{\infty\}$ denotes the Fredholm index of the corresponding isometric Bogoliubov operator $V$. As shown by Longo [14],

*Supported by the Deutsche Forschungsgemeinschaft (Sfb 288 "Differentialgeometrie und Quantenphysik")

${ }^{\dagger} \mathrm{E}$-mail: binnenhe@physik.fu-berlin.de 
an analogous result holds in the theory of superselection sectors where the statistical dimension of a localized endomorphism coincides with the square root of the Jones index of the associated inclusion of local algebras.

We derive explicit formulae for the implementing isometries (i.e. for an orthonormal basis of the implementing Hilbert space) based on the work of Ruijsenaars 8. For this purpose, we generalize the definition of Ruijsenaars' operator $\Lambda$ (called the 'associate' of a given Bogoliubov operator) and obtain one implementing isometry $\Psi_{0}$ in terms of the Wick ordered exponential of the unbounded bilinear Hamiltonian induced by $\Lambda$. A complete set of implementing isometries may then be constructed by multiplying $\Psi_{0}$ with suitable partial isometries. In this way, the implementing Hilbert space itself acquires a Fock space structure, with $\Psi_{0}$ playing the role of the vacuum.

The set of Bogoliubov operators $V$ fulfilling the Shale-Stinespring condition (for a fixed Fock representation) forms a topological semigroup w.r.t. a suitably chosen metric. By a result of Araki [15], the subgroup of unitaries (ind $V=0$ ) consists of two connected components. We prove by contrary that each subset of Bogoliubov operators with fixed non-vanishing Fredholm index is connected.

Our interest in implementable Bogoliubov endomorphisms originates from the speculation that they might serve to construct localized endomorphisms for free Fermi fields with non-abelian gauge groups [16]. We intend to discuss this idea in a subsequent paper. It should be mentioned that Bogoliubov transformations have been successfully used in the construction of localized endomorphisms in conformal field theory models [17, 18, 19].

This article is organized as follows. CAR algebras, Bogoliubov transformations and quasi-free states are introduced in Section 2. Throughout the paper Araki's formalism of selfdual CAR algebras [20, 12, 15] is used which is equivalent to the more familiar notion of complexified Clifford algebras over real Hilbert spaces [21. However, Araki's approach has the advantage of being complex-linear from the beginning. The usual description of a CAR algebra by means of annihilation and creation operators enters through Fock representations of the selfdual CAR algebra. In this section, we also compute Watatani indices of inclusions that are induced by arbitrary Bogoliubov endomorphisms.

Implementability of endomorphisms of $\mathrm{C}^{*}$-algebras is defined in Section 3.1. We shortly discuss uniqueness of implementing operators and indices of associated inclusions. Then we turn to CAR algebras and Bogoliubov endomorphisms. We describe the decomposition of $\pi \circ \varrho$ into cyclic subrepresentations where $\pi$ is a Fock representation and $\varrho$ a Bogoliubov endomorphism. The already mentioned PowersStørmer-Araki criterion then enables us to prove the validity of the Shale-Stinespring condition in the general case. We have included a new proof of a recent result of Böckenhauer [22] (decomposition of $\pi \circ \varrho$ into irreducibles) in Section 3.2 since we consider our proof to have some interest on its own. We show that $\pi \circ \varrho$ is equivalent to a multiple of either a Fock representation or a direct sum of two inequivalent pseudo Fock representations, depending on the index.

Section 1 contains the main result of our investigation, namely the detailed construction of a complete set of implementers for a given implementable endomorphism. In Section 4.1, Wick ordered unbounded bilinear Hamiltonians and their Wick ordered exponentials are defined in a representation-dependent way with the help of unsmeared annihilation and creation operators. Then commutation relations of these exponentials with annihilation and creation operators are computed. The associate $\Lambda$ is characterized by intertwining properties of the corresponding exponential, but is not unique. A complete set of implementing isometries is defined in Section 4.2. As a key to the proof of completeness, we present a decomposition of $\varrho$ into a product of two simpler transformations in Section 4.3. This product decomposition also leads to an interesting decomposition of implementers.

Finally, we prove the aforementioned result on connectedness in Section 5 . Our argumentation parallels in part the reasoning of Carey, Hurst and O'Brien in [23] and relies on the product decomposition developed in Section 4.3 . 


\section{Preliminaries}

Let $\mathcal{K}$ be an infinite-dimensional complex Hilbert space ${ }^{a}$ with a fixed conjugation (i.e. antiunitary involution) $\Gamma$, and let $\mathfrak{B}(\mathcal{K})$ be the algebra of bounded linear operators on $\mathcal{K}$. For $A \in \mathfrak{B}(\mathcal{K})$ we set

$$
\bar{A}:=\Gamma A \Gamma .
$$

Let $\mathcal{C}_{0}(\mathcal{K}, \Gamma)$ be the ${ }^{*}$-algebra, unique up to isomorphism, which is algebraically generated by the range of a linear embedding $B: \mathcal{K} \rightarrow \mathcal{C}_{0}(\mathcal{K}, \Gamma)$ with relations

$$
\begin{aligned}
& B(k)^{*}=B(\Gamma k), \\
& \left\{B(k)^{*}, B\left(k^{\prime}\right)\right\}=\left\langle k, k^{\prime}\right\rangle \mathbf{1}, \quad k, k^{\prime} \in \mathcal{K} .
\end{aligned}
$$

Here $\{$,$\} denotes the anticommutator. \mathcal{C}_{0}(\mathcal{K}, \Gamma)$ is just the (complexified) Clifford algebra [21, 24] over the real Hilbert space $\operatorname{Re} \mathcal{K}:=\{k \in \mathcal{K} \mid \Gamma k=k\}$; conversely, given a real Hilbert space, one may recover $\mathcal{K}, \Gamma$ (and $B$ ) by complexification (details are in [16]). There is a unique $\mathrm{C}^{*}$-norm on $\mathcal{C}_{0}(\mathcal{K}, \Gamma)$ (which fulfills $\left.\|B(k)\|^{2}=\frac{1}{2}\left(\|k\|^{2}+\left(\|k\|^{4}-|\langle k, \Gamma k\rangle|^{2}\right)^{1 / 2}\right)\right)$, and completion in this norm yields a simple $\mathrm{C}^{*}$-algebra $\mathcal{C}(\mathcal{K}, \Gamma)$, namely Araki's selfdual $C A R$ algebra over $(\mathcal{K}, \Gamma)$ [20, 12, 15].

Bogoliubov transformations are precisely the unital ${ }^{*}$-endomorphisms of $\mathcal{C}(\mathcal{K}, \Gamma)$ that leave $\mathcal{K}$ invariant. Put differently, every isometry $V \in \mathfrak{B}(\mathcal{K})$ that commutes with $\Gamma$ (and therefore restricts to a real-linear isometry of $\operatorname{Re} \mathcal{K})$ induces a unital, isometric *-endomorphism $\varrho_{V}$ of $\mathcal{C}(\mathcal{K}, \Gamma)$ through

$$
\varrho_{V}(B(k))=B(V k), \quad k \in \mathcal{K} .
$$

Such isometries are called Bogoliubov operators, and the semigroup of Bogoliubov operators is denoted by

$$
\mathcal{I}(\mathcal{K}, \Gamma):=\left\{V \in \mathfrak{B}(\mathcal{K}) \mid V^{*} V=\mathbf{1}, \bar{V}=V\right\} .
$$

The map $V \mapsto \varrho_{V}$ is a unital isomorphism from $\mathcal{I}(\mathcal{K}, \Gamma)$ onto the semigroup of Bogoliubov endomorphisms; for fixed $A \in \mathcal{C}(\mathcal{K}, \Gamma)$, the map $V \mapsto \varrho_{V}(A)$ is continuous w.r.t. strong topology on $\mathcal{I}(\mathcal{K}, \Gamma)$ and norm topology on $\mathcal{C}(\mathcal{K}, \Gamma)$.

Let $V \in \mathcal{I}(\mathcal{K}, \Gamma)$. Since $\operatorname{ran} V$ is closed and $\operatorname{ker} V=\{0\}, V$ and $V^{*}$ are semi-Fredholm operators in the sense of Kato 25] and have well-defined Fredholm indices. The map

$$
\mathcal{I}(\mathcal{K}, \Gamma) \rightarrow \mathbb{N} \cup\{\infty\}, \quad V \mapsto \operatorname{ind} V^{*}=-\operatorname{ind} V=\operatorname{dim} \operatorname{ker} V^{*}
$$

is a surjective homomorphism of semigroups $(0 \in \mathbb{N}$ by convention). Hence $\mathcal{I}(\mathcal{K}, \Gamma)$ is the disjoint union of subsets

$$
\mathcal{I}(\mathcal{K}, \Gamma)=\bigcup_{n \in \mathbb{N} \cup\{\infty\}} \mathcal{I}^{n}(\mathcal{K}, \Gamma), \quad \mathcal{I}^{n}(\mathcal{K}, \Gamma):=\left\{V \in \mathcal{I}(\mathcal{K}, \Gamma) \mid \text { ind } V^{*}=n\right\} .
$$

Note that $\varrho_{V}$ is an automorphism if and only if $V \in \mathcal{I}^{0}(\mathcal{K}, \Gamma)$, the group of unitary Bogoliubov operators, in which case we prefer to use the symbol " $\alpha$ " instead of " $\varrho$ ". For $V_{1}, V_{2} \in \mathcal{I}^{n}(\mathcal{K}, \Gamma)$ there exists $U \in \mathcal{I}^{0}(\mathcal{K}, \Gamma)$ with $V_{1}=U V_{2}$. Such $U$ has the form $U=V_{1} V_{2}^{*}+u$ where $u$ is a partial isometry with $(\operatorname{ker} u)^{\perp}=\operatorname{ker} V_{2}^{*}, \operatorname{ran} u=\operatorname{ker} V_{1}^{*}$, and $u=\bar{u}$. We may express this in a more sophisticated way by saying that $\mathcal{I}^{0}(\mathcal{K}, \Gamma)$ acts on $\mathcal{I}(\mathcal{K}, \Gamma)$ by left multiplication, that the orbits of this action are just the sets $\mathcal{I}^{n}(\mathcal{K}, \Gamma)$, and that the stabilizer of $V \in \mathcal{I}^{n}(\mathcal{K}, \Gamma)$ is isomorphic to $O(n)$ (the orthogonal group of an $n$-dimensional real Hilbert space).

${ }^{a}$ We are solely dealing with separable Hilbert spaces in this article. 
Next we describe the set of states we are interested in. A state $\omega$ over $\mathcal{C}(\mathcal{K}, \Gamma)$ is called quasi-free [12] if its $\mathrm{n}$-point functions have the form

$$
\begin{aligned}
\omega\left(B\left(k_{1}\right) \cdots B\left(k_{2 m+1}\right)\right) & =0, \\
\omega\left(B\left(k_{1}\right) \cdots B\left(k_{2 m}\right)\right) & =(-1)^{\frac{m(m-1)}{2}} \sum_{\sigma} \operatorname{sign} \sigma \omega\left(B\left(k_{\sigma(1)}\right) B\left(k_{\sigma(m+1)}\right)\right) \cdots \omega\left(B\left(k_{\sigma(m)}\right) B\left(k_{\sigma(2 m)}\right)\right)
\end{aligned}
$$

where the sum runs over all permutations $\sigma$ satisfying $\sigma(1)<\ldots<\sigma(m)$ and $\sigma(j)<\sigma(j+m), j=$ $1, \ldots, m$. Therefore quasi-free states are completely determined by their two-point functions, and we have a bijection between the convex set

$$
\mathcal{Q}(\mathcal{K}, \Gamma):=\{S \in \mathfrak{B}(\mathcal{K}) \mid 0 \leq S \leq \mathbf{1}, \bar{S}=\mathbf{1}-S\}
$$

and the (non-convex) set of quasi-free states given by

$$
S \mapsto \omega_{S}, \quad \omega_{S}\left(B(k)^{*} B\left(k^{\prime}\right)\right)=\left\langle k, S k^{\prime}\right\rangle .
$$

The following lemma is immediate.

Lemma 2.1 The semigroup of Bogoliubov endomorphisms acts from the right on the set of quasi-free states by $\omega \mapsto \omega \circ \varrho, \mathcal{I}(\mathcal{K}, \Gamma)$ acts from the right on $\mathcal{Q}(\mathcal{K}, \Gamma)$ by $S \mapsto V^{*} S V$, and

$$
\omega_{S} \circ \varrho_{V}=\omega_{V * S V} .
$$

Projections in $\mathcal{Q}(\mathcal{K}, \Gamma)$ are called basis projections and the corresponding states Fock states; the latter are precisely the pure quasi-free states [26]. The group of Bogoliubov automorphisms acts transitively on the set of Fock states as $\mathcal{I}^{0}(\mathcal{K}, \Gamma)$ acts transitively on the set of basis projections. Note that for a basis projection $P$, the complementary (basis) projection is simply given by $\bar{P}$. Since $\omega_{P}\left(B(k)^{*} B(k)\right)=0$ if $k \in \bar{P}(\mathcal{K})$, the elements of $B(\bar{P}(\mathcal{K}))$ (resp. $B(P(\mathcal{K}))$ ) correspond to annihilation (resp. creation) operators in the state $\omega_{P}$. A (faithful and irreducible) GNS representation $\pi_{P}$ for $\omega_{P}$ is given by

$$
\pi_{P}(B(k)):=a(P k)^{*}+a(P \Gamma k)
$$

on the antisymmetric Fock space $\mathcal{F}_{a}(P(\mathcal{K}))$ over $P(\mathcal{K})$ with the usual Fock vacuum $\Omega_{P}$ as cyclic vector and annihilation operators $a(f), f \in P(\mathcal{K})$. In a Fock representation $\pi_{P}$, a Bogoliubov endomorphism $\varrho_{V}$ induces the transformation

$$
a(f) \mapsto a_{V}(f):=a(P V P f)+a(P V \bar{P} \Gamma f)^{*}, \quad f \in P(\mathcal{K}),
$$

which shows the connection to the (state-dependent) description of Bogoliubov transformations by pairs of operators $(P V P, P V \bar{P} \Gamma)$ as preferred by some authors (e.g. [27]).

Given a basis projection $P$, a state over $\mathcal{C}(\mathcal{K}, \Gamma)$ is said to be gauge invariant if it is invariant under the one-parameter group of Bogoliubov automorphisms $\left(\alpha_{U_{\lambda}}\right)_{\lambda \in \mathbb{R}}$ with $U_{\lambda}:=e^{i \lambda} P+e^{-i \lambda} \bar{P} \in \mathcal{I}^{0}(\mathcal{K}, \Gamma)$. As follows from Lemma 2.1, a quasi-free state $\omega_{S}$ is gauge invariant if and only if $[P, S]=0$.

The so-called central state $\omega_{1 / 2}$ [21, 24, 12 is the unique tracial state over $\mathcal{C}(\mathcal{K}, \Gamma)$. By uniqueness, $\omega_{1 / 2}$ is invariant under all unital ${ }^{*}$-endomorphisms of $\mathcal{C}(\mathcal{K}, \Gamma)$.

Now suppose we have an orthogonal decomposition $\mathcal{K}=\mathcal{K}_{1} \oplus \mathcal{K}_{2}$ into $\Gamma$-invariant closed subspaces with $\mathcal{K}_{2}$ finite dimensional]. Set $\Gamma_{j}:=\left.\Gamma\right|_{\mathcal{K}_{j}}$ and regard $\mathcal{C}\left(\mathcal{K}_{j}, \Gamma_{j}\right), j=1,2$ as subalgebras of $\mathcal{C}(\mathcal{K}, \Gamma)$.

\footnotetext{
${ }^{b}$ If $\operatorname{dim} \mathcal{K}_{2}$ is odd, then $\mathcal{C}\left(\mathcal{K}_{2}, \Gamma_{2}\right)$ is not uniquely determined by $(\mathbb{1})$; in addition, one requires it to have non-trivial center (see [15).
} 
Then $\mathcal{C}(\mathcal{K}, \Gamma)$ is canonically isomorphic to the $\mathbb{Z}_{2}$-graded tensor product of $\mathcal{C}\left(\mathcal{K}_{1}, \Gamma_{1}\right)$ and $\mathcal{C}\left(\mathcal{K}_{2}, \Gamma_{2}\right)$ [28] through identification of $A_{1} \otimes A_{2}$ with $A_{1} \cdot A_{2} \in \mathcal{C}(\mathcal{K}, \Gamma), A_{j} \in \mathcal{C}\left(\mathcal{K}_{j}, \Gamma_{j}\right)$ (here $\mathbb{Z}_{2}=\{0,1\}$, and the grading is induced by $\alpha_{-1}$, i.e. $\left.\mathcal{C}(\mathcal{K}, \Gamma)=\mathcal{C}(\mathcal{K}, \Gamma)_{0} \oplus \mathcal{C}(\mathcal{K}, \Gamma)_{1}, \mathcal{C}(\mathcal{K}, \Gamma)_{g}:=\left\{A \mid \alpha_{-1}(A)=(-1)^{g} A\right\}\right)$. Hence all elements of $\mathcal{C}(\mathcal{K}, \Gamma)$ are finite sums of elements $A_{1} A_{2}$ as above, and we have a well-defined linear mapping

$$
E: \mathcal{C}(\mathcal{K}, \Gamma) \rightarrow \mathcal{C}\left(\mathcal{K}_{1}, \Gamma_{1}\right), \quad A_{1} A_{2} \mapsto A_{1} \omega_{1 / 2}\left(A_{2}\right), \quad A_{j} \in \mathcal{C}\left(\mathcal{K}_{j}, \Gamma_{j}\right)
$$

Lemma 2.2 $E$ is a faithful conditional expectation from $\mathcal{C}(\mathcal{K}, \Gamma)$ onto $\mathcal{C}\left(\mathcal{K}_{1}, \Gamma_{1}\right)$ with Watatani index

$$
\operatorname{index} E=\operatorname{dim} \mathcal{C}\left(\mathcal{K}_{2}, \Gamma_{2}\right)=2^{\operatorname{dim} \mathcal{K}_{2}} .
$$

Proof. We first show $E\left(A^{*}\right)=E(A)^{*}, A \in \mathcal{C}(\mathcal{K}, \Gamma)$. By linearity, it suffices to check this for elements of the form $A=A_{1} A_{2}$ with $A_{j} \in \mathcal{C}\left(\mathcal{K}_{j}, \Gamma_{j}\right)$ homogeneous. By use of the anticommutation relations,

$$
\begin{aligned}
E\left(A^{*}\right) & =(-1)^{\operatorname{deg} A_{1} \operatorname{deg} A_{2}} E\left(A_{1}^{*} A_{2}^{*}\right) \\
& =(-1)^{\operatorname{deg} A_{1} \operatorname{deg} A_{2}} A_{1}^{*} \omega_{1 / 2}\left(A_{2}^{*}\right) \\
& =A_{1}^{*} \overline{\omega_{1 / 2}\left(A_{2}\right)} \quad\left(\text { since } \operatorname{deg} A_{2} \neq 0 \text { implies } \omega_{1 / 2}\left(A_{2}^{*}\right)=0\right) \\
& =E(A)^{*} .
\end{aligned}
$$

Hence $E$ is positive. Now let $A, B_{1}, C \in \mathcal{C}\left(\mathcal{K}_{1}, \Gamma_{1}\right)$ and $B_{2} \in \mathcal{C}\left(\mathcal{K}_{2}, \Gamma_{2}\right)$ be given, with $B_{2}$ and $C$ homogeneous. Then

$$
\begin{aligned}
E\left(A B_{1} B_{2} C\right) & =(-1)^{\operatorname{deg} B_{2} \operatorname{deg} C} E\left(A B_{1} C B_{2}\right) \\
& =(-1)^{\operatorname{deg} B_{2} \operatorname{deg} C} A B_{1} C \omega_{1 / 2}\left(B_{2}\right) \\
& =A E\left(B_{1} B_{2}\right) C .
\end{aligned}
$$

By linearity, $E(A B C)=A E(B) C$ for $A, C \in \mathcal{C}\left(\mathcal{K}_{1}, \Gamma_{1}\right), B \in \mathcal{C}(\mathcal{K}, \Gamma)$, so $E$ is a conditional expectation.

To compute the Watatani index [13] of $E$ we need a 'quasi-basis', i.e. a finite subset $\left\{B_{\beta}\right\} \subset \mathcal{C}(\mathcal{K}, \Gamma)$ fulfilling

$$
\sum_{\beta} E\left(A B_{\beta}\right) B_{\beta}^{*}=A, \quad A \in \mathcal{C}(\mathcal{K}, \Gamma)
$$

index $E$ is then defined as index $E:=\sum_{\beta} B_{\beta} B_{\beta}^{*}$ and does not depend on the choice of quasi-basis. The existence of a quasi-basis also guarantees faithfulness of $E$.

Here we may obtain a quasi-basis as follows. Let $\left\{b_{1}, \ldots, b_{n}\right\}$ be an orthonormal basis for $\mathcal{K}_{2}$ consisting of $\Gamma$-invariant vectors $\left(n<\infty\right.$ by assumption). Let $I_{n}$ denote the set of $2^{n}$ multi-indices $\beta=\left(\beta_{1}, \ldots, \beta_{l}\right)$ obeying

$$
0 \leq l \leq n, \quad 1 \leq \beta_{1}<\ldots<\beta_{l} \leq n \quad(\beta:=0 \text { for } l=0) .
$$

Set $B_{j}:=\sqrt{2} B\left(b_{j}\right)$ for $j=1, \ldots, n$ and $B_{\beta}:=B_{\beta_{1}} \cdots B_{\beta_{l}}$ for $\beta \in I_{n}\left(B_{0}:=\mathbf{1}\right)$.

We claim that $\left(B_{\beta}\right)_{\beta \in I_{n}}$ is a quasi-basis for $E$ (by construction, it is a basis for $\mathcal{C}\left(\mathcal{K}_{2}, \Gamma_{2}\right)$ ). Note that $\left\{B_{j}, B_{m}\right\}=2 \delta_{j m} \mathbf{1}, j, m=1, \ldots, n$, and $B_{\beta}^{*}=(-1)^{l(l-1) / 2} B_{\beta}$ if $\beta=\left(\beta_{1}, \ldots, \beta_{l}\right) \in I_{n}$. Furthermore $\omega_{1 / 2}\left(B_{\beta}\right)=\delta_{\beta 0}$ 21, 12, hence $\omega_{1 / 2}\left(B_{\beta}^{*} B_{\gamma}\right)=\delta_{\beta \gamma}$. Again by linearity, it suffices to consider elements of the form $A=A_{1} B_{\beta}^{*}, A_{1} \in \mathcal{C}\left(\mathcal{K}_{1}, \Gamma_{1}\right), \beta \in I_{n}$. We have

$$
\sum_{\gamma \in I_{n}} E\left(A B_{\gamma}\right) B_{\gamma}^{*}=A_{1} \sum_{\gamma \in I_{n}} \omega_{1 / 2}\left(B_{\beta}^{*} B_{\gamma}\right) B_{\gamma}^{*}=A_{1} B_{\beta}^{*}=A .
$$


Therefore $\left(B_{\beta}\right)_{\beta \in I_{n}}$ is a quasi-basis for $E$, and using $B_{\beta} B_{\beta}^{*}=\mathbf{1}$ we get

$$
\text { index } E=\sum_{\beta \in I_{n}} B_{\beta} B_{\beta}^{*}=2^{n} \mathbf{1}, \quad n=\operatorname{dim} \mathcal{K}_{2} .
$$

Next we show that $E$ is the conditional expectation with minimal index, so the index of the inclusion of simple $\mathrm{C}^{*}$-algebras $\mathcal{C}\left(\mathcal{K}_{1}, \Gamma_{1}\right) \subset \mathcal{C}(\mathcal{K}, \Gamma)$ equals

$$
\left[\mathcal{C}(\mathcal{K}, \Gamma): \mathcal{C}\left(\mathcal{K}_{1}, \Gamma_{1}\right)\right]=\operatorname{index} E=2^{\operatorname{dim} \mathcal{K}_{1}^{\perp}} .
$$

Lemma 2.3 $E$ is the unique minimal conditional expectation from $\mathcal{C}(\mathcal{K}, \Gamma)$ onto $\mathcal{C}\left(\mathcal{K}_{1}, \Gamma_{1}\right)$.

Proof. Following Watatani [13] we have to show

$$
\text { index } E \cdot E(A)=\sum_{\beta \in I_{n}} B_{\beta} A B_{\beta}^{*}
$$

for $A \in \mathcal{C}\left(\mathcal{K}_{1}, \Gamma_{1}\right)^{c}$, the $\mathrm{C}^{*}$-algebra of elements of $\mathcal{C}(\mathcal{K}, \Gamma)$ that commute with all elements of $\mathcal{C}\left(\mathcal{K}_{1}, \Gamma_{1}\right)$. We claim that $\mathcal{C}\left(\mathcal{K}_{1}, \Gamma_{1}\right)^{c}$ equals $\mathcal{C}\left(\mathcal{K}_{2}, \Gamma_{2}\right)_{0}$, the even subalgebra of $\mathcal{C}\left(\mathcal{K}_{2}, \Gamma_{2}\right)$. Indeed, writing $A=\sum_{\beta} A_{\beta} B_{\beta}$ with $A_{\beta} \in \mathcal{C}\left(\mathcal{K}_{1}, \Gamma_{1}\right)$, all $A_{\beta}$ have to commute with the elements of $\mathcal{C}\left(\mathcal{K}_{1}, \Gamma_{1}\right)_{0}$. Let $P$ be a basis projection of $\left(\mathcal{K}_{1}, \Gamma_{1}\right)$ and $\Psi(-\mathbf{1})$ a unitary implementing $\alpha_{-1}$ in $\pi_{P}$ (which exists due to invariance of $\omega_{P}$ under $\alpha_{-1}$ and is unique up to a phase) then $\pi_{P}\left(\mathcal{C}\left(\mathcal{K}_{1}, \Gamma_{1}\right)_{0}\right)^{\prime \prime}=\{\Psi(-\mathbf{1})\}^{\prime}$. It follows that $\pi_{P}\left(A_{\beta}\right) \in \pi_{P}\left(\mathcal{C}\left(\mathcal{K}_{1}, \Gamma_{1}\right)_{0}\right)^{\prime}=\{\Psi(-\mathbf{1})\}^{\prime \prime}=\operatorname{span}\{\mathbf{1}, \Psi(-\mathbf{1})\}$. But since $\alpha_{-1}$ is not inner [1, 12, 15], we have $\Psi(-1) \notin \pi_{P}\left(\mathcal{C}\left(\mathcal{K}_{1}, \Gamma_{1}\right)\right)$. Thus $A_{\beta} \in \mathbb{C} 1$ and $A \in \mathcal{C}\left(\mathcal{K}_{2}, \Gamma_{2}\right)_{0}$.

It suffices to prove (的) for $A=B_{\gamma}, \gamma=\left(\gamma_{1}, \ldots, \gamma_{l}\right) \in I_{n}, l$ even (the case $A=\mathbf{1}$ is clear by definition of index $E$ ). In the following computation we use the notation $\beta \cap \gamma:=\left\{\beta_{1}, \ldots, \beta_{r}\right\} \cap\left\{\gamma_{1}, \ldots, \gamma_{l}\right\}$ if $\beta=\left(\beta_{1}, \ldots, \beta_{r}\right) \in I_{n} . \quad \beta^{\prime} \in I_{n}$ will then denote the multi-index whose entries are the elements of $\left\{\beta_{1}, \ldots, \beta_{r}\right\} \backslash(\beta \cap \gamma)$.

$$
\begin{aligned}
\sum_{\beta \in I_{n}} B_{\beta} A B_{\beta}^{*} & =\sum_{m=0}^{l} \sum_{1 \leq j_{1}<\ldots<j_{m} \leq l} \sum_{\substack{\beta, \beta \cap \gamma=\\
\left\{\gamma_{j_{1}}, \ldots, \gamma_{j_{m}}\right\}}} B_{\beta} B_{\gamma} B_{\beta}^{*} \\
& =\sum_{m=0}^{l} \sum_{j_{1}<\ldots<j_{m}} \sum_{\substack{\beta \cap \gamma=\\
\left\{\gamma_{j_{1}}, \ldots, \gamma_{j_{m}}\right\}}} B_{\beta^{\prime}} B_{\gamma_{j_{1}}} \cdots B_{\gamma_{j_{m}}} B_{\gamma}\left(B_{\gamma_{j_{1}}} \cdots B_{\gamma_{j_{m}}}\right)^{*} B_{\beta^{\prime}}^{*} \\
& =\sum_{m=0}^{l} \sum_{j_{1}<\ldots<j_{m}} \sum_{\substack{\beta \cap \gamma=\\
\left\{\gamma_{j_{1}}, \cdots, \gamma_{j_{m}}\right\}}}(-1)^{m} B_{\gamma} \underbrace{B_{\beta^{\prime}} B_{\beta^{\prime}}^{*}}_{\mathbf{1}} \underbrace{B_{\gamma_{j_{1}}} \cdots B_{\gamma_{j_{m}}}\left(B_{\gamma_{j_{1}}} \cdots B_{\gamma_{j_{m}}}\right)^{*}}_{\mathbf{1}} \\
& =B_{\gamma} \sum_{m=0}^{l}(-1)^{m}\left(\begin{array}{c}
l \\
m
\end{array}\right) \cdot 2^{n-l}=2^{n-l} B_{\gamma}(-1+1)^{l}=0 .
\end{aligned}
$$

But we also have $E\left(B_{\gamma}\right)=\omega_{1 / 2}\left(B_{\gamma}\right)=0$ if $\gamma \neq 0$. 
Let us return to Bogoliubov transformations. The possible ranges of Bogoliubov operators are just the infinite-dimensional $\Gamma$-invariant closed subspaces of $\mathcal{K}$, and for $V \in \mathcal{I}(\mathcal{K}, \Gamma)$, we may identify $\varrho_{V}(\mathcal{C}(\mathcal{K}, \Gamma))$ with $\mathcal{C}\left(\operatorname{ran} V,\left.\Gamma\right|_{\operatorname{ran} V}\right)$. Thus we have just seen that

$$
\left[\mathcal{C}(\mathcal{K}, \Gamma): \varrho_{V}(\mathcal{C}(\mathcal{K}, \Gamma))\right]=2^{\text {ind } V^{*}}
$$

if ind $V^{*}<\infty$, and this causes us to assign to each Bogoliubov operator a number

$$
d_{V}:=2^{\frac{1}{2} \text { ind } V^{*}} \leq \infty, \quad V \in \mathcal{I}(\mathcal{K}, \Gamma)
$$

analogous to the statistical dimension in the theory of superselection sectors [14]. $d$ is obviously multiplicative

$$
d_{V V^{\prime}}=d_{V} d_{V^{\prime}}
$$

Note that $d_{V}$ is defined without reference to any representation, but if $\varrho_{V}$ happens to be implementable in a Fock representation, then $d_{V}$ shows up as the dimension of the implementing Hilbert space. More generally, we shall see in Section 3.2 that the representations $\pi_{P} \circ \varrho_{V}$ (with $P$ a basis projection and $V$ an arbitrary Bogoliubov operator) split into $d_{V}$ resp. $\sqrt{2} d_{V}$ irreducibles if ind $V^{*}$ is even resp. odd (cf. [22]). Also note that the conditional expectations $E$ defined above allow the definition of left inverses $[10] \varrho^{-1} \circ E$ for Bogoliubov endomorphisms. More explicitly, for a Bogoliubov endomorphism $\varrho_{V}$, a left inverse $\Phi_{V}$ is given by $\Phi_{V}\left(A_{1} A_{2}\right):=\varrho_{V}^{-1}\left(A_{1}\right) \omega_{1 / 2}\left(A_{2}\right)$ if $A_{j} \in \mathcal{C}\left(\mathcal{K}_{j}, \Gamma_{j}\right), \mathcal{K}_{1}:=\operatorname{ran} V, \mathcal{K}_{2}:=\operatorname{ker} V^{*}$.

An essential ingredient for our analysis in Section 3 will be the criterion for quasi-equivalence of quasifree states as derived by Powers and Størmer [11] for gauge invariant states and generalized by Araki [12]. By definition, two states $\omega, \omega^{\prime}$ are quasi-equivalent (denoted by " $\approx$ ") if they induce quasi-equivalent GNS-representations. Now let $\mathfrak{J}_{p}(\mathcal{K})$ be the trace ideal

$$
\mathfrak{J}_{p}(\mathcal{K}):=\left\{A \in \mathfrak{B}(\mathcal{K}) \mid\|A\|_{p}<\infty\right\}, \quad 1 \leq p<\infty
$$

with trace norm $\|A\|_{p}:=\left(\operatorname{tr}\left(|A|^{p}\right)\right)^{1 / p}$, and let $S, S^{\prime} \in \mathcal{Q}(\mathcal{K}, \Gamma)$. The statement is

$$
\omega_{S} \approx \omega_{S^{\prime}} \Longleftrightarrow S^{1 / 2}-S^{1 / 2} \in \mathfrak{J}_{2}(\mathcal{K}) .
$$

It has been observed by Powers [29] that this criterion may be simplified if one of the operators $S, S^{\prime}$ is a projection. Namely, if $P$ is a basis projection, then

$$
\omega_{P} \approx \omega_{S} \Longleftrightarrow \bar{P} S \bar{P} \in \mathfrak{J}_{1}(\mathcal{K}) .
$$

\section{Implementability and Equivalence of Representations}

The famous result of Shale and Stinespring [1] asserts that a Bogoliubov automorphism $\alpha_{V}, V \in \mathcal{I}^{0}(\mathcal{K}, \Gamma)$, is unitarily implementable in a Fock representation $\pi_{P}$ if and only if

$$
[P, V] \in \mathfrak{J}_{2}(\mathcal{K}) .
$$

'Unitarily implementable' stands for the existence of a unitary operator $\Psi$ on Fock space fulfilling Ad $\Psi \circ$ $\pi_{P}=\pi_{P} \circ \alpha_{V}$ where

$$
(\operatorname{Ad} \Psi)(X):=\Psi X \Psi^{*}
$$

(in the following, we shall use the notation $\operatorname{Ad} \Psi$ also for partially isometric $\Psi$ ). Note that the ShaleStinespring condition immediately follows from (8) (or (9)). In fact, existence of $\Psi$ is equivalent to quasiequivalence of the irreducible representations $\pi_{P}$ and $\pi_{P} \circ \alpha_{V}$. Since $\pi_{P} \circ \alpha_{V}$ is a GNS-representation for 
$\omega_{P} \circ \alpha_{V}=\omega_{V^{*} P V}$ (see Lemma 2.1), $\pi_{P} \approx \pi_{P} \circ \alpha_{V}$ if and only if $P-V^{*} P V=V^{*}[V, P] \in \mathfrak{J}_{2}(\mathcal{K}$ ) by (8) (remember that $P$ and $V^{*} P V$ are projections).

We shall show first that an endomorphism $\varrho_{V}$ is implementable in a Fock representation $\pi_{P}$ (in an appropriate sense) if and only if (10) holds. Later we shall study the action of the group of implementable automorphisms on the semigroup of endomorphisms (with finite index). This will lead us to a description of equivalence classes of representations $\pi_{P} \circ \varrho_{V}$.

\subsection{Implementability of Endomorphisms}

To generalize the notion of implementability to the case of endomorphisms we adopt ideas of Doplicher and Roberts [9]. The unitary implementer $\Psi$ above gets thereby replaced by a set of isometries fulfilling the relations of a Cuntz algebra [30]. We give a definition for arbitrary $\mathrm{C}^{*}$-algebras.

Definition 3.1 $A{ }^{*}$-endomorphism $\varrho$ of a $C^{*}$-algebra $\mathfrak{A}$ is (isometrically) implementable in a representation $(\pi, \mathcal{H})$ if there exists a (possibly finite) sequence $\left(\Psi_{n}\right)_{n \in I}$ in $\mathfrak{B}(\mathcal{H})$ with relations

$$
\Psi_{m}^{*} \Psi_{n}=\delta_{m n} \mathbf{1}, \quad \sum_{n \in I} \Psi_{n} \Psi_{n}^{*}=\mathbf{1}^{c},
$$

which implements @ by

$$
\pi \circ \varrho=\sum_{n \in I} \operatorname{Ad} \Psi_{n} \circ \pi^{c} .
$$

$\mathcal{H}$ then decomposes into the orthogonal direct sum of the ranges of the isometries $\Psi_{n}$, and $\pi \circ \varrho$ decomposes into subrepresentations $\left.\pi \circ \varrho\right|_{\operatorname{ran} \Psi_{n}}$, each of them unitarily equivalent to $\pi$. But the converse is also true, i.e. $\varrho$ is implementable in $\pi$ if and only if $\pi \circ \varrho$ is equivalent to a multiple of $\pi$. For irreducible $\pi$ this reads

$$
\varrho \text { is implementable in } \pi \Longleftrightarrow \pi \circ \varrho \approx \pi \text {. }
$$

By (11), we may regard the implementing isometries $\left(\Psi_{n}\right)_{n \in I}$ as an orthonormal basis of the Hilbert space $H:=\overline{\operatorname{span}\left(\Psi_{n}\right)}$ in $\mathfrak{B}(\mathcal{H})$ with scalar product given by $\Psi^{*} \Psi^{\prime}=\left\langle\Psi, \Psi^{\prime}\right\rangle \mathbf{1}$ (this scalar product induces the usual operator norm). Every element $\Psi$ of $H$ is an intertwiner from $\pi$ to $\pi \circ \varrho$ :

$$
\Psi \pi(A)=\pi(\varrho(A)) \Psi, \quad A \in \mathfrak{A} .
$$

Note that $H$ coincides with the space of intertwiners from $\pi$ to $\pi \circ \varrho$ if and only if $\pi$ is irreducible. If $\pi$ is reducible, there may exist several Hilbert spaces implementing $\varrho$, mutually related by unitaries in $\pi(\varrho(\mathfrak{A}))^{\prime}$. More precisely, if $\left(\Psi_{n}\right)_{n \in I}$ and $\left(\Psi_{n}^{\prime}\right)_{n \in I}$ both implement $\varrho$ in $\pi$ (we may choose the same index sets), then $\Psi:=\sum_{n} \Psi_{n}^{\prime} \Psi_{n}^{*}$ is a unitary in $\pi(\varrho(\mathfrak{A}))^{\prime}$, and $\Psi_{n}^{\prime}=\Psi \Psi_{n}$. Conversely, given $\left(\Psi_{n}\right)_{n \in I}$ and a unitary $\Psi \in \pi(\varrho(\mathfrak{A}))^{\prime},\left(\Psi \Psi_{n}\right)_{n \in I}$ is a set of implementing isometries (cf. [31).

An implementable endomorphism $\varrho$ gives rise to normal ${ }^{*}$-endomorphisms $\varrho_{H}:=\sum_{n \in I} \operatorname{Ad} \Psi_{n}$ of $\mathfrak{B}(\mathcal{H})$, and one finds 14.

$$
\left[\mathfrak{B}(\mathcal{H}): \varrho_{H}(\mathfrak{B}(\mathcal{H}))\right]=d_{\varrho}^{2}
$$

where $d_{\varrho}:=\operatorname{dim} H$ does not depend on the choice of $H=\overline{\operatorname{span}\left(\Psi_{n}\right)}$. Let us outline the computation of the index in the setting of Watatani (cf. the proofs of Lemmas 2.2, 2.3) for the case $d_{\varrho}<\infty$. $\Phi_{H}:=$ $d_{\varrho}^{-1} \sum_{n} \operatorname{Ad} \Psi_{n}^{*}$ is a left inverse for $\varrho_{H}$ yielding the conditional expectation $E_{H}:=\varrho_{H} \circ \Phi_{H}$ from $\mathfrak{B}(\mathcal{H})$ onto $\varrho_{H}(\mathfrak{B}(\mathcal{H})) .\left(\sqrt{d_{\varrho}} \Psi_{n}^{*}\right)_{n=1, \ldots, d_{\varrho}}$ is a quasi-basis (cf. (四)) for $E_{H}$, hence index $E_{H}=d_{\varrho} \sum_{n} \Psi_{n}^{*} \Psi_{n}=$ $\underline{d_{\varrho}^{2}}$. To show minimality of $E_{H}$, one must check (6) $d_{\varrho} E_{H}(A)=\sum_{l} \Psi_{l}^{*} A \Psi_{l}$ for $A \in \varrho_{H}(\mathfrak{B}(\mathcal{H}))^{\prime}$. But

\footnotetext{
${ }^{c}$ w.r.t. strong topology if $I$ is infinite
} 
$\varrho_{H}(\mathfrak{B}(\mathcal{H}))^{\prime}=\operatorname{span}\left\{\Psi \Psi^{\prime *} \mid \Psi, \Psi^{\prime} \in H\right\} \cong \mathfrak{B}(H)$, and $d_{\varrho} E_{H}\left(\Psi_{m} \Psi_{n}^{*}\right)=\delta_{m n} \mathbf{1}=\sum_{l} \Psi_{l}^{*}\left(\Psi_{m} \Psi_{n}^{*}\right) \Psi_{l}$. Thus $E_{H}$ is minimal and $\left[\mathfrak{B}(\mathcal{H}): \varrho_{H}(\mathfrak{B}(\mathcal{H}))\right]=d_{\varrho}^{2}$.

We shall show in Section 1 that $d_{\varrho_{V}}=d_{V}$ (defined by (7)) if $\varrho_{V}$ is a Bogoliubov endomorphism, implementable in some Fock representation.

Let us add a last remark on the general situation. Suppose we are given a set of implementers $\left(\Psi_{n}\right)_{n \in I}$. Then for $m, n \in I, \Psi_{m} \Psi_{n}^{*} \in \pi(\varrho(\mathfrak{A}))^{\prime}$ is a partial isometry containing $\operatorname{ran} \Psi_{n}$ in its initial space, and $\Psi_{m}=\left(\Psi_{m} \Psi_{n}^{*}\right) \Psi_{n}$. This suggests to construct a complete set of implementing isometries by multiplying one isometry $\Psi$ fulfilling (14) with certain partial isometries in $\pi(\varrho(\mathfrak{A}))^{\prime}$. We shall employ this idea in Section 4.2 .

After this digression we concentrate on Bogoliubov transformations again. Inspection of (13) leads us to study the representations $\pi_{P} \varrho_{V}$; as will turn out, they are quasi-equivalent to GNS-representations associated with the states $\omega_{P} \varrho_{V}$ (a similar observation has been made, in a different setting, by Rideau [32]). To see this let $P$ be a basis projection and $V \in \mathcal{I}(\mathcal{K}, \Gamma)$, and regard

$$
v:=P V V^{*} P
$$

as an operator on $P(\mathcal{K})$. The direct sum decomposition $P(\mathcal{K})=\operatorname{ker} v \oplus \overline{\text { ran } v}$ induces a tensor product decomposition of Fock space: $\mathcal{F}_{a}(P(\mathcal{K})) \cong \mathcal{F}_{a}(\operatorname{ker} v) \otimes \mathcal{F}_{a}(\overline{\operatorname{ran} v})$. Choose an orthonormal basis $\left(f_{j}\right)_{j=1, \ldots, N_{V}}$ for $\operatorname{ker} v$ where

$$
N_{V}:=\operatorname{dim} \operatorname{ker} v \leq \frac{1}{2} \text { ind } V^{*}
$$

(the inequality follows from $\operatorname{ker} v \oplus \Gamma \operatorname{ker} v \subset \operatorname{ker} V^{*}$, and set $A(f):=a(f) \Psi(-\mathbf{1})$ with a unitary $\Psi(-\mathbf{1})$ implementing $\alpha_{-1}$ in $\pi_{P}$ (cf. the proof of Lemma 2.3). Let $I_{N_{V}}$ be the set of multi-indices $\beta=\left(\beta_{1}, \ldots, \beta_{l}\right)$ as in (5) (with finite entries $\beta_{j}$ ) and define

$$
\begin{aligned}
& A_{\beta}:=A\left(f_{\beta_{1}}\right) \cdots A\left(f_{\beta_{l}}\right) \quad\left(A_{0}:=\mathbf{1}\right) \\
& \phi_{\beta}^{V}:=A_{\beta}^{*} \Omega_{P}, \\
& \mathcal{F}_{\beta}^{V}:=\pi_{P}\left(\varrho_{V}(\mathcal{C}(\mathcal{K}, \Gamma))\right) \phi_{\beta}^{V} \\
& \pi_{\beta}^{V}:=\left.\pi_{P} \circ \varrho_{V}\right|_{\mathcal{F}_{\beta}^{V}} ^{V} .
\end{aligned}
$$

Note that the $A_{\beta}$ are partial isometries in $\pi_{P}\left(\varrho_{V}(\mathcal{C}(\mathcal{K}, \Gamma))\right)^{\prime}$.

Lemma 3.2 Each of the $2^{N_{V}}$ cyclic subrepresentations $\left(\pi_{\beta}^{V}, \mathcal{F}_{\beta}^{V}, \phi_{\beta}^{V}\right)$ induces the state $\omega_{P} \varrho_{V}$, and $\pi_{P} \varrho_{V}$ splits into their direct sum: $\pi_{P} \circ \varrho_{V}=\bigoplus_{\beta \in I_{N_{V}}} \pi_{\beta}^{V}$.

Proof. Invariance of $\mathcal{F}_{\beta}^{V}$ and cyclicity of $\phi_{\beta}^{V}$ are clear by definition. Since $A_{\beta} \in \pi_{P}\left(\varrho_{V}(\mathcal{C}(\mathcal{K}, \Gamma))\right)^{\prime}$ and $A_{\beta} A_{\beta}^{*} \Omega_{P}=\Omega_{P}$, we have $\left\langle\phi_{\beta}^{V}, \pi_{\beta}^{V}(A) \phi_{\beta}^{V}\right\rangle=\left\langle\Omega_{P}, \pi_{P}\left(\varrho_{V}(A)\right) \Omega_{P}\right\rangle=\omega_{P}\left(\varrho_{V}(A)\right), A \in \mathcal{C}(\mathcal{K}, \Gamma)$. Thus $\left(\pi_{\beta}^{V}, \mathcal{F}_{\beta}^{V}, \phi_{\beta}^{V}\right)$ is a GNS-triple for $\omega_{P} \circ \varrho_{V}$ (and the representations $\pi_{\beta}^{V}$ are mutually unitarily equivalent).

Next we show $\mathcal{F}_{\beta}^{V} \perp \mathcal{F}_{\gamma}^{V}$ for $\beta \neq \gamma$. Since at least one of the vectors $A_{\beta} A_{\gamma}^{*} \Omega_{P}, A_{\gamma} A_{\beta}^{*} \Omega_{P}$ vanishes if $\beta \neq \gamma$, we have for $A, B \in \mathcal{C}(\mathcal{K}, \Gamma)$

$$
\left\langle\pi_{P}\left(\varrho_{V}(A)\right) \phi_{\beta}^{V}, \pi_{P}\left(\varrho_{V}(B)\right) \phi_{\gamma}^{V}\right\rangle=\left\langle A_{\beta}^{*} \Omega_{P}, \pi_{P}\left(\varrho_{V}\left(A^{*} B\right)\right) A_{\gamma}^{*} \Omega_{P}\right\rangle=0,
$$

implying orthogonality of $\mathcal{F}_{\beta}^{V}$ and $\mathcal{F}_{\gamma}^{V}$.

Finally we have to prove $\mathcal{F}_{a}(P(\mathcal{K}))=\bigoplus_{\beta} \mathcal{F}_{\beta}^{V}$. Using $\pi_{P}\left(\varrho_{V}(B(k))\right)=a(P V k)^{*}+a(P V \Gamma k), k \in \mathcal{K}$, one can show by induction on the particle number

$$
\mathcal{F}_{0}^{V}=\overline{\pi_{P}\left(\varrho_{V}(\mathcal{C}(\mathcal{K}, \Gamma))\right) \Omega_{P}}=\mathcal{F}_{a}(\overline{\operatorname{ran} P V})=\mathcal{F}_{a}(\overline{\operatorname{ran} v}) .
$$


Since the $\phi_{\beta}^{V}$ form an orthonormal basis for $\mathcal{F}_{a}(\operatorname{ker} v)$, the assertion follows.

The decomposition of these cyclic representations into irreducibles will be examined in Section 3.2. First we state the main result of this section. Remember that $\bar{P}=\mathbf{1}-P$.

Theorem 3.3 A Bogoliubov endomorphism $\varrho_{V}$ is isometrically implementable in a Fock representation $\pi_{P}$ if and only if $P V \bar{P} \in \mathfrak{J}_{2}(\mathcal{K})$.

Proof. In view of (13) and Lemma 3.2, $\varrho_{V}$ is implementable in $\pi_{P}$ if and only if $\omega_{P} \circ \varrho_{V} \approx \omega_{P}$. Lemma 2.1 and the Powers-Størmer-Araki criterion in the form (9) imply that $\omega_{P} \varrho_{V} \approx \omega_{P}$ if and only if $\bar{P} V^{*} P V \bar{P} \in$ $\mathfrak{J}_{1}(\mathcal{K})$. The latter condition is clearly equivalent to $P V \bar{P} \in \mathfrak{J}_{2}(\mathcal{K})$.

Note that $P V \bar{P}$ is Hilbert-Schmidt if and only if $[P, V]=P V \bar{P}-\bar{P} V P$ is, so the Shale-Stinespring condition (10) remains valid. We denote the semigroup of Bogoliubov operators fulfilling (10) by

$$
\mathcal{I}_{P}(\mathcal{K}, \Gamma):=\left\{V \in \mathcal{I}(\mathcal{K}, \Gamma) \mid P V \bar{P} \in \mathfrak{J}_{2}(\mathcal{K})\right\} .
$$

Since $P V \bar{P}$ and $\bar{P} V P$ are compact for $V \in \mathcal{I}_{P}(\mathcal{K}, \Gamma),(P V P) \oplus(\bar{P} V \bar{P})=V-P V \bar{P}-\bar{P} V P$ is semiFredholm, and

$$
\text { ind } V^{*}=2 \text { ind } P V^{*} P \in 2 \mathbb{N} \cup\{\infty\}
$$

(we used $\bar{P} V \bar{P}=\Gamma(P V P) \Gamma)$. Thus we have a decomposition (cf. (2))

$$
\mathcal{I}_{P}(\mathcal{K}, \Gamma)=\bigcup_{m \in \mathbb{N} \cup\{\infty\}} \mathcal{I}_{P}^{2 m}(\mathcal{K}, \Gamma), \quad \mathcal{I}_{P}^{2 m}(\mathcal{K}, \Gamma):=\mathcal{I}_{P}(\mathcal{K}, \Gamma) \cap \mathcal{I}^{2 m}(\mathcal{K}, \Gamma) .
$$

In particular, the "statistical dimension" $d_{V}$ defined by $(7)$ is contained in $\mathbb{N} \cup\{\infty\}$ if $V \in \mathcal{I}_{P}(\mathcal{K}, \Gamma)$. Let us finally remark that non-surjective Bogoliubov endomorphisms cannot be inner since $\mathcal{C}(\mathcal{K}, \Gamma)$, being $\mathrm{AF}$ and thus finite, does not contain non-unitary isometries.

\subsection{Equivalence of Representations}

As mentioned in Section 2, the $\mathcal{I}^{0}(\mathcal{K}, \Gamma)$-orbits in $\mathcal{I}(\mathcal{K}, \Gamma)$ w.r.t. left multiplication are just the subsets $\mathcal{I}^{n}(\mathcal{K}, \Gamma)$. In the present section, we are interested in $\mathcal{I}_{P}^{0}(\mathcal{K}, \Gamma)$-orbits (for fixed $P$ ) since each such orbit gives rise to a unique equivalence class of representations $\pi_{P} \circ \varrho_{V}$. For $V \in \mathcal{I}(\mathcal{K}, \Gamma)$, we use the notation

$$
S_{V}:=V^{*} P V \in \mathcal{Q}(\mathcal{K}, \Gamma), \quad Q_{V}:=\mathbf{1}-V V^{*},
$$

and the symbol ' $\simeq$ ' will mean 'unitarily equivalent'. We only consider the action of $\mathcal{I}_{P}^{0}(\mathcal{K}, \Gamma)$ on the semigroup of Bogoliubov operators with finite index

$$
\mathcal{I}^{\mathrm{fin}}(\mathcal{K}, \Gamma):=\left\{V \in \mathcal{I}(\mathcal{K}, \Gamma) \mid \text { ind } V^{*}<\infty\right\} .
$$

For $V \in \mathcal{I}^{\text {fin }}(\mathcal{K}, \Gamma)$, the operators $Q_{V}$ (the projection onto ker $V^{*}$ ) and $S_{V} \overline{S_{V}}=-V^{*} P Q_{V} \bar{P} V$ have finite rank.

Lemma 3.4 Let $V, V^{\prime} \in \mathcal{I}^{\text {fin }}(\mathcal{K}, \Gamma)$. Then the following conditions are equivalent:

a) $\pi_{P} \circ \varrho_{V}$ and $\pi_{P} \circ \varrho_{V}$ are unitarily equivalent;

b) there exists $U \in \mathcal{I}_{P}^{0}(\mathcal{K}, \Gamma)$ with $V^{\prime}=U V$;

c) ind $V=$ ind $V^{\prime}$, and $S_{V}-S_{V^{\prime}}$ is Hilbert-Schmidt. 
Proof. We first show a) $\Rightarrow$ c). By Lemma 3.2, $\pi_{P} \circ \varrho_{V} \simeq \pi_{P} \circ \varrho_{V^{\prime}}$ implies $\omega_{S_{V}}=\omega_{P} \circ \varrho_{V} \approx \omega_{P^{\circ}} \varrho_{V^{\prime}}=\omega_{S_{V^{\prime}}}$.

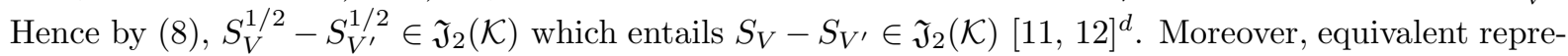
sentations have isomorphic commutants. We have (cf. [12] $\pi_{P}\left(\varrho_{V}(\mathcal{C}(\mathcal{K}, \Gamma))\right)^{\prime}=\left(\pi_{P}\left(B\left(\operatorname{ker} V^{*}\right)\right) \Psi(-\mathbf{1})\right)^{\prime \prime}$ with $\Psi(-\mathbf{1})$ as in the proof of Lemma 2.3. Hence the commutants have dimensions $2^{\text {ind } V^{*}}$ resp. $2^{\text {ind } V^{\prime *}}$, and the indices of $V$ and $V^{\prime}$ must be equal.

Next we show c) $\Rightarrow$ b). Let $u$ be a partial isometry with initial space $\operatorname{ker} V^{*}$, final space $\operatorname{ker} V^{\prime *}$, and $u=\bar{u}$ (such $u$ exists due to $\Gamma$-invariance and equality of dimensions of the kernels). Then $U:=V^{\prime} V^{*}+u$ is an element of $\mathcal{I}^{0}(\mathcal{K}, \Gamma)$ and fulfills $V^{\prime}=U V$. We have to prove that $P U \bar{P} \in \mathfrak{J}_{2}(\mathcal{K})$. But $u$ has finite rank, so it suffices to show

$$
A:=\bar{P} V S_{V^{\prime}} V^{*} \bar{P} \in \mathfrak{J}_{1}(\mathcal{K}) .
$$

Since $S_{V} \overline{S_{V}}$ and $S_{V^{\prime}} \overline{S_{V^{\prime}}}$ have finite rank, $S_{V^{\prime}} \overline{S_{V}}+S_{V} \overline{S_{V^{\prime}}}=\left(S_{V^{\prime}}-S_{V}\right)\left(\overline{S_{V}}-\overline{S_{V^{\prime}}}\right)+S_{V} \overline{S_{V}}+S_{V^{\prime}} \overline{S_{V^{\prime}}}$ is trace class. But the same is true for $A=A Q_{V}+A V V^{*}=A Q_{V}+\bar{P} V\left(S_{V^{\prime}} \overline{S_{V}}+S_{V} \overline{S_{V^{\prime}}}\right) V^{*}+\bar{P} Q_{V} P V \overline{S_{V^{\prime}}} V^{*}$.

b) $\Rightarrow$ a) is obvious.

In order to make use of part c) of the lemma, we need information about the operators $S_{V}$. An orthogonal projection $E$ on $\mathcal{K}$ is called a partial basis projection 12 if $E \bar{E}=0$. By definition, the $\Gamma$-codimension of $E$ is the dimension of $\operatorname{ker}(E+\bar{E})$. The following lemma holds for arbitrary $S \in \mathcal{Q}(\mathcal{K}, \Gamma)$ (except for the formula for the $\Gamma$-codimension, of course) as long as $S \bar{S}$ has finite rank.

Lemma 3.5 Let $V \in \mathcal{I}^{\text {fin }}(\mathcal{K}, \Gamma)$ and let $E_{V}$ denote the orthogonal projection onto $\operatorname{ker} S_{V} \overline{S_{V}}$. Then $S_{V} E_{V}=E_{V} S_{V}$ is a partial basis projection with finite $\Gamma$-codimension ind $V^{*}-2 N_{V}$. Moreover, there exist $\lambda_{1}, \ldots, \lambda_{r} \in\left(0, \frac{1}{2}\right)$, partial basis projections $E_{1}, \ldots, E_{r}$ and an orthogonal projection $E_{\frac{1}{2}}=\overline{E_{\frac{1}{2}}}$ such that $E_{V}+E_{\frac{1}{2}}+\sum_{j=1}^{r}\left(E_{j}+\overline{E_{j}}\right)=\mathbf{1}$ and

$$
S_{V}=S_{V} E_{V}+\frac{1}{2} E_{\frac{1}{2}}+\sum_{j=1}^{r}\left(\lambda_{j} E_{j}+\left(1-\lambda_{j}\right) \overline{E_{j}}\right) .
$$

Proof. Since $S_{V} \overline{S_{V}}=S_{V}-S_{V}^{2}, S_{V}$ commutes with $E_{V}$ and fulfills $S_{V} E_{V}=S_{V}^{2} E_{V}$ and also $\left(S_{V} E_{V}\right)\left(\Gamma S_{V} E_{V} \Gamma\right)=S_{V} \overline{S_{V}} E_{V}=0$. Hence $S_{V} E_{V}$ is a partial basis projection. The dimension of $\operatorname{ker}\left(S_{V} E_{V}+\overline{S_{V} E_{V}}\right)=\operatorname{ker} E_{V}$ (the $\Gamma$-codimension of $S_{V} E_{V}$ ) equals the rank of $S_{V} \overline{S_{V}}$ which is finite for $V \in \mathcal{I}^{\text {fin }}(\mathcal{K}, \Gamma)$. By $S_{V} \overline{S_{V}}=V^{*} P Q_{V} P V$, the rank of $S_{V} \overline{S_{V}}$ equals $\operatorname{dim} V^{*} P\left(\operatorname{ker} V^{*}\right)$. Now consider the decomposition

$$
\operatorname{ker} V^{*}=\operatorname{ker} v \oplus \operatorname{ker} \bar{v} \oplus\left(\operatorname{ker} V^{*} \ominus(\operatorname{ker} v \oplus \operatorname{ker} \bar{v})\right)
$$

with $v$ given by (15). $V^{*} P$ vanishes on $\operatorname{ker} v \oplus \operatorname{ker} \bar{v}$, but the restriction of $V^{*} P$ to $\operatorname{ker} V^{*} \ominus(\operatorname{ker} v \oplus \operatorname{ker} \bar{v})$ is one-to-one since $V^{*} P k=0=V^{*} k$ implies $V^{*} \bar{P} k=0$, i.e. $k \in \operatorname{ker} v \oplus \operatorname{ker} \bar{v}$. Hence the $\Gamma$-codimension of $S_{V} E_{V}$ equals $\operatorname{dim}\left(\operatorname{ker} V^{*} \ominus(\operatorname{ker} v \oplus \operatorname{ker} \bar{v})\right)=\operatorname{ind} V^{*}-2 N_{V}$.

Let $s_{V}$ denote the restriction of $S_{V}$ to $\operatorname{ran} S_{V} \overline{S_{V}} \cdot s_{V}$ is a positive operator on a finite dimensional Hilbert space and has a complete set of eigenvectors with eigenvalues in $(0,1)$. If $\lambda$ is an eigenvalue of $s_{V}$, then $1-\lambda$ is also an eigenvalue (with the same multiplicity) due to $s_{V}+\overline{s_{V}}=\mathbf{1}-E_{V}$. Thus there exist $\lambda_{1}, \ldots, \lambda_{r} \in\left(0, \frac{1}{2}\right)$ and spectral projections $E_{\frac{1}{2}}, E_{1}, \ldots, E_{r}$ with $\overline{E_{\frac{1}{2}}}=E_{\frac{1}{2}}, E_{j} \overline{E_{j}}=0$ such that $E_{\frac{1}{2}}+\sum_{j=1}^{r}\left(E_{j}+\overline{E_{j}}\right)=\mathbf{1}-E_{V}$ and $s_{V}=\frac{1}{2} E_{\frac{1}{2}}+\sum_{j=1}^{r}\left(\lambda_{j} E_{j}+\left(1-\lambda_{j}\right) E_{j}\right)$.

As a consequence, operators $S_{V}$ with ind $V^{*}=1$ necessarily have the form $S_{V}=S_{V} E_{V}+\frac{1}{2} E_{\frac{1}{2}}$ where

\footnotetext{
${ }^{d}$ By an argument in [19], the conditions $S_{V}^{1 / 2}-S_{V^{\prime}}^{1 / 2} \in \mathfrak{J}_{2}(\mathcal{K})$ and $S_{V}-S_{V^{\prime}} \in \mathfrak{J}_{2}(\mathcal{K})$ are actually equivalent for $V, V^{\prime} \in \mathcal{I}^{\mathrm{fin}}(\mathcal{K}, \Gamma)$.
} 
$E_{\frac{1}{2}}=1-E_{V}$ has rank one. By taking direct sums of $V \in \mathcal{I}^{1}(\mathcal{K}, \Gamma)$ with operators $V(\varphi)$ from the example below, we see that each combination of eigenvalues and multiplicities that is allowed by Lemma 3.5 actually occurs for some $S_{V^{\prime}}$. We further remark that a quasi-free state $\omega_{S}$ with $S$ of the form (18) is a product state 月 $^{2}$ as defined by Powers [33 (see also 26, 24]) w.r.t. the decomposition $\mathcal{K}=\operatorname{ker} S \bar{S} \oplus \operatorname{ran} E_{\frac{1}{2}} \oplus \bigoplus_{j} \operatorname{ran}\left(E_{j}+\overline{E_{j}}\right)$. Clearly, the restriction of $\omega_{S}$ to $\mathcal{C}\left(\operatorname{ker} S \bar{S},\left.\Gamma\right|_{\operatorname{ker} S \bar{S}}\right)$ is a Fock state, the restriction to $\mathcal{C}\left(\operatorname{ran} E_{\frac{1}{2}},\left.\Gamma\right|_{\operatorname{ran} E_{\frac{1}{2}}}\right)$ the central state.

Example. Let $\left(f_{n}\right)_{n \in \mathbb{N}}$ be an orthonormal basis for $P(\mathcal{K})$ and set $E_{n}:=f_{n}\left\langle f_{n},.\right\rangle, f_{n}^{+}:=\left(f_{n}+\right.$ $\left.\Gamma f_{n}\right) / \sqrt{2}, f_{n}^{-}:=i\left(f_{n}-\Gamma f_{n}\right) / \sqrt{2}$. Then $\left(f_{n}^{s}\right)_{s= \pm, n \in \mathbb{N}}$ is an orthonormal basis for $\mathcal{K}$ consisting of $\Gamma-$ invariant vectors. For $\varphi \in \mathbb{R}$, define a Bogoliubov operator

$$
V(\varphi):=\left(f_{0}^{+} \cos \varphi+f_{1}^{-} \sin \varphi\right)\left\langle f_{0}^{+}, .\right\rangle+\left(f_{0}^{-} \sin \varphi-f_{1}^{+} \cos \varphi\right)\left\langle f_{0}^{-}, .\right\rangle+\sum_{s= \pm, n \geq 1} f_{n+1}^{s}\left\langle f_{n}^{s}, .\right\rangle .
$$

Then $V(\varphi) \in \mathcal{I}^{2}(\mathcal{K}, \Gamma)$, and the eigenvalue $\lambda_{\varphi}=(1+\sin 2 \varphi) / 2$ of $S_{V(\varphi)}=\left(\lambda_{\varphi} E_{0}+\left(1-\lambda_{\varphi}\right) \overline{E_{0}}\right)+\sum_{n \geq 1} E_{n}$ assumes any value in $[0,1]$ as $\varphi$ varies over $[-\pi / 4, \pi / 4]$.

Next we characterize the Bogoliubov operators $V$ for which $S_{V}$ takes a particularly simple form. A distinction arises between the cases of even and odd Fredholm index.

Lemma 3.6 a) Let $W \in \mathcal{I}(\mathcal{K}, \Gamma)$. Then the following conditions are equivalent:

(i) $\omega_{P} \circ \varrho_{W}$ is a pure state;

(ii) $S_{W}$ is a basis projection;

(iii) $\left[P, W W^{*}\right]=0$.

If any of these conditions is fulfilled, then ind $W^{*}=2 N_{W}$ and $\pi_{P} \circ \varrho_{W} \simeq d_{W} \cdot \pi_{S_{W}}$.

b) For any basis projection $P^{\prime}$ and $m \in \mathbb{N} \cup\{\infty\}$, there exists $W \in \mathcal{I}^{2 m}(\mathcal{K}, \Gamma)$ with $S_{W}=P^{\prime}$.

c) Let $W \in \mathcal{I}^{\mathrm{fin}}(\mathcal{K}, \Gamma)$. Then the following conditions are equivalent:

(i) $\omega_{P} \circ \varrho_{W}$ is a mixture of two disjoint pure states;

(ii) $S_{W} E_{W}$ is a partial basis projection with $\Gamma$-codimension 1;

(iii) $\left[P, W W^{*}\right]$ has rank 2;

(iv) ind $W^{*}=2 N_{W}+1$.

d) For any partial basis projection $P^{\prime}$ with $\Gamma$-codimension 1 and $m \in \mathbb{N} \cup\{\infty\}$, there exists $W \in$ $\mathcal{I}^{2 m+1}(\mathcal{K}, \Gamma)$ with $S_{W} E_{W}=P^{\prime}$.

Proof. a) We know from Section 2 that $\omega_{P} \circ \varrho_{W}$ is pure if and only if $S_{W}$ is a projection. We have

$$
S_{W}^{2}=S_{W} \Longleftrightarrow W^{*} P Q_{W} P W=0 \Longleftrightarrow Q_{W} P W W^{*}=0 \Longleftrightarrow\left[P, W W^{*}\right]=0 .
$$

If this is fulfilled, $\operatorname{ker} W W^{*}=\operatorname{ker}\left(P W W^{*} P\right) \oplus \operatorname{ker}\left(\bar{P} W W^{*} \bar{P}\right)$ has dimension $2 N_{W}$. By Lemma 3.2, $\pi_{P \circ \varrho_{W}}$ is the direct sum of $d_{W}=2^{N_{W}}$ irreducible subrepresentations, each equivalent to the Fock representation $\pi_{S_{W}}$.

b) Let $m$ and $P^{\prime}$ be given. There clearly exists $W^{\prime} \in \mathcal{I}^{2 m}(\mathcal{K}, \Gamma)$ with $\left[P, W^{\prime}\right]=0$. Since $\mathcal{I}^{0}(\mathcal{K}, \Gamma)$ acts transitively on the set of basis projections, we may choose $U \in \mathcal{I}^{0}(\mathcal{K}, \Gamma)$ with $U^{*} P U=P^{\prime}$. Then $W:=W^{\prime} U$ has the desired properties.

c) (ii) $\Leftrightarrow$ (iii) follows from the facts that the $\Gamma$-codimension of $S_{W} E_{W}$ equals the rank of $W W^{*} P Q_{W}$ (cf. the proof of Lemma 3.5) and that $\left[P, W W^{*}\right]=Q_{W} P W W^{*}-W W^{*} P Q_{W}$. (ii) and (iv) are equivalent

${ }^{e}$ A state $\omega$ is a product state w.r.t. a decomposition $\mathcal{K}=\oplus_{j} \mathcal{K}_{j}$ of $\mathcal{K}$ into closed, $\Gamma$-invariant subspaces if $\omega(A B)=$ $\omega(A) \omega(B)$ whenever $A \in \mathcal{C}\left(\mathcal{K}_{j},\left.\Gamma\right|_{\mathcal{K}_{j}}\right), B \in \mathcal{C}\left(\mathcal{K}_{j}^{\perp},\left.\Gamma\right|_{\mathcal{K}_{j}^{\perp}}\right)$. In this case, the restrictions $\omega_{j}$ of $\omega$ to $\mathcal{C}\left(\mathcal{K}_{j}, \Gamma \mid \mathcal{K}_{j}\right)$ are even (i.e. invariant under $\alpha_{-1}$ ) with at most one exception. If all $\omega_{j}$ are even, then $\omega$ is pure if and only if all $\omega_{j}$ are 33 . 
by virtue of Lemma 3.5. (ii) $\Rightarrow$ (i) has been shown by Araki [12]. To prove (i) $\Rightarrow$ (iv), assume that ind $W^{*}-2 N_{W}>1$ (if ind $W^{*}=2 N_{W}$, then $S_{W}$ is a basis projection and $\omega_{P} \circ \varrho_{W}$ pure). By Lemma 3.5, there exist a two-dimensional, $\Gamma$-invariant subspace $\mathcal{K}_{1} \subset \mathcal{K}, \lambda \in(0,1)$ and a basis projection $E$ of $\left(\mathcal{K}_{1}, \Gamma_{1}\right), \Gamma_{1}:=\left.\Gamma\right|_{\mathcal{K}_{1}}$, such that $S_{1}:=\left.S\right|_{\mathcal{K}_{1}}=\lambda E+(1-\lambda) \bar{E}$. Set $\mathcal{K}_{2}:=\mathcal{K}_{1}^{\perp}, \Gamma_{2}:=\left.\Gamma\right|_{\mathcal{K}_{2}}$ and $S_{2}:=\left.S\right|_{\mathcal{K}_{2}}$. Then $\omega_{S}$ is a product state w.r.t. $\mathcal{K}=\mathcal{K}_{1} \oplus \mathcal{K}_{2}$; we write $\omega_{S}=\omega_{S_{1}} \otimes \omega_{S_{2}}$ which means that $\omega_{S}\left(A_{1} A_{2}\right)=$ $\omega_{S_{1}}\left(A_{1}\right) \omega_{S_{2}}\left(A_{2}\right), A_{j} \in \mathcal{C}\left(\mathcal{K}_{j}, \Gamma_{j}\right)$. As $\omega_{S_{1}}=\lambda \omega_{E}+(1-\lambda) \omega_{\bar{E}}$ is a mixture of two equivalent Fock states over $\mathcal{C}\left(\mathcal{K}_{1}, \Gamma_{1}\right), \omega_{S}=\lambda\left(\omega_{E} \otimes \omega_{S_{2}}\right)+(1-\lambda)\left(\omega_{\bar{E}} \otimes \omega_{S_{2}}\right)=\lambda \omega_{E+S_{2}}+(1-\lambda) \omega_{\bar{E}+S_{2}}$ is a mixture of two quasi-equivalent quasi-free states over $\mathcal{C}(\mathcal{K}, \Gamma)$. We are going to show that $\omega_{E} \otimes \omega_{S_{2}}$ and $\omega_{\bar{E}} \otimes \omega_{S_{2}}$ are orthogonal. It is readily seen that $\omega_{S_{1}}$ is faithful. Let $\left(\pi_{1}, \mathcal{H}_{1}, \Omega_{1}\right)$ be the GNS-representation for $\omega_{S_{1}}$, i.e. $\mathcal{H}_{1}=\mathcal{C}\left(\mathcal{K}_{1}, \Gamma_{1}\right)$ as a vector space, $\Omega_{1}=1, \pi_{1}$ acts by left multiplication, and $\omega_{S_{1}}(A)=\left\langle\Omega_{1}, \pi_{1}(A) \Omega_{1}\right\rangle$. Since $\omega_{S_{1}}$ is even, we may implement $\alpha_{-1}$ by the self-adjoint unitary $\Psi_{1}(\mathbf{- 1})$ with $\Psi_{1}(-\mathbf{1}) \pi_{1}(A) \Omega_{1}=$ $\pi_{1}\left(\alpha_{-1}(A)\right) \Omega_{1}$. Now choose a unit vector $e \in E\left(\mathcal{K}_{1}\right)$, set $e^{+}:=(e+\Gamma e) / \sqrt{2}, e^{-}:=i(e-\Gamma e) / \sqrt{2}$, and define complementary orthogonal projections $P^{ \pm}:=\frac{1}{2} \mathbf{1} \pm i \pi_{1}\left(B\left(e^{-}\right) B\left(e^{+}\right)\right) \Psi_{1}(-\mathbf{1}) \in \pi_{1}\left(\mathcal{C}\left(\mathcal{K}_{1}, \Gamma_{1}\right)\right)^{\prime}$. A computation shows

$$
\left\langle\Omega_{1}, \pi_{1}(A) P^{+} \Omega_{1}\right\rangle=\lambda \omega_{E}(A), \quad\left\langle\Omega_{1}, \pi_{1}(A) P^{-} \Omega_{1}\right\rangle=(1-\lambda) \omega_{\bar{E}}(A), \quad A \in \mathcal{C}\left(\mathcal{K}_{1}, \Gamma_{1}\right) .
$$

Let $\left(\pi_{2}, \mathcal{H}_{2}, \Omega_{2}\right)$ be the GNS-representation for $\omega_{S_{2}}$. Then the GNS-representation $\left(\pi_{S}, \mathcal{H}_{S}, \Omega_{S}\right)$ for $\omega_{S}$ may be identified with the $\mathbb{Z}_{2}$-graded tensor product of $\pi_{1}$ and $\pi_{2}$. Since $\operatorname{deg} P^{ \pm}=0$ and $P^{ \pm} \in \pi_{1}\left(\mathcal{C}\left(\mathcal{K}_{1}, \Gamma_{1}\right)\right)^{\prime}$, the projections $P^{ \pm} \otimes \mathbf{1}$ lie in the commutant $\pi_{S}(\mathcal{C}(\mathcal{K}, \Gamma))^{\prime}$. We now infer from $\left\langle\Omega_{S}, \pi_{S}(A)\left(P^{+} \otimes \mathbf{1}\right) \Omega_{S}\right\rangle=\lambda\left(\omega_{E} \otimes \omega_{S_{2}}\right)(A)$ and $\left\langle\Omega_{S}, \pi_{S}(A)\left(P^{-} \otimes \mathbf{1}\right) \Omega_{S}\right\rangle=(1-\lambda)\left(\omega_{\bar{E}} \otimes \omega_{S_{2}}\right)(A)$ that $\omega_{E} \otimes \omega_{S_{2}}$ and $\omega_{\bar{E}} \otimes \omega_{S_{2}}$ are indeed orthogonal. Hence $\omega_{S}$ cannot be a mixture of two disjoint pure states. This proves (i) $\Rightarrow$ (iv) and therefore part c).

d) Let $\left(f_{n}\right)_{n \in \mathbb{N}}$ be an orthonormal basis for $P(\mathcal{K}),\left(g_{n}\right)_{n \geq 1}$ an orthonormal basis for $P^{\prime}(\mathcal{K})$, and $g_{0}$ a unit vector in $\operatorname{ker}\left(P^{\prime}+\overline{P^{\prime}}\right)$. Set $V:=f_{0}^{+}\left\langle g_{0},.\right\rangle+\sum_{s= \pm, n \geq 1} f_{n}^{s}\left\langle g_{n}^{s},.\right\rangle$ (we use the notation of the example). Then $V \in \mathcal{I}^{1}(\mathcal{K}, \Gamma)$ and $S_{V}=\frac{1}{2} g_{0}\left\langle g_{0},.\right\rangle+P^{\prime}$. This implies $S_{V} E_{V}=P^{\prime}$, and if we choose $W^{\prime}$ as in the proof of b), then $W:=W^{\prime} V$ has the desired properties.

One may use the argument given in the proof of c) inductively to show that a quasi-free state $\omega_{S}$ with $S$ of the form (18) is a mixture of $2^{m}$ mutually orthogonal, pure states if the rank of $S \bar{S}$ is $2 m$ or $2 m-1$.

Now let us discuss the decomposition of representations $\pi_{P} \circ \varrho_{V}$ with $V \in \mathcal{I}^{\text {fin }}(\mathcal{K}, \Gamma)$. If ind $V^{*}$ is even (resp. odd), then $S_{V} E_{V}$ is a partial basis projection with even (odd) $\Gamma$-codimension by Lemma 3.5, and there exists a basis projection (partial basis projection with $\Gamma$-codimension 1) $P^{\prime}$ with $P^{\prime}-S_{V} \in \mathfrak{J}_{2}(\mathcal{K})$ (we may choose $P^{\prime}$ to coincide with $S_{V} E_{V}$ on ker $S_{V} \overline{S_{V}}$; then $P^{\prime}-S_{V}$ has finite rank). By Lemma 3.6, there exists $W$ with ind $W=$ ind $V$ and $S_{W} E_{W}=P^{\prime}$, and Lemma 3.4 implies $\pi_{P} \circ \varrho_{V} \simeq \pi_{P} \circ \varrho_{W}$. The latter representation splits into $2^{N_{W}}$ copies of the GNS-representation $\pi_{S_{W}}$ for the state $\omega_{P} \circ \varrho_{W}$ by Lemma 3.2. If ind $V^{*}$ is even, $\pi_{S_{W}}=\pi_{P^{\prime}}$ and $2^{N_{W}}=d_{V}$. If ind $V^{*}$ is odd, then $\pi_{S_{W}}=\pi^{+} \oplus \pi^{-}$where $\pi^{ \pm}$are mutually inequivalent, irreducible, so-called pseudo Fock representations by virtue of a lemma of Araki (see [12] for details), and $2^{N_{W}}=2^{-1 / 2} d_{V}$.

Summarizing, we rediscover Böckenhauer's result [22]:

Theorem 3.7 Let $P$ be a basis projection and $V \in \mathcal{I}^{\text {fin }}(\mathcal{K}, \Gamma)$. If ind $V^{*}$ is even, then there exist basis projections $P^{\prime}$ with $P^{\prime}-S_{V} \in \mathfrak{J}_{2}(\mathcal{K})$, and for each such $P^{\prime}$

$$
\pi_{P} \circ \varrho_{V} \simeq d_{V} \cdot \pi_{P^{\prime}} .
$$

If ind $V^{*}$ is odd, there exist partial basis projections $P^{\prime}$ with $\Gamma$-codimension 1 and $P^{\prime}-S_{V} \in \mathfrak{J}_{2}(\mathcal{K})$. For each such $P^{\prime}$,

$$
\pi_{P} \circ \varrho_{V} \simeq 2^{-1 / 2} d_{V} \cdot\left(\pi_{P^{\prime}}^{+} \oplus \pi_{P^{\prime}}^{-}\right)
$$

where $\pi_{P^{\prime}}^{ \pm}$are the (inequivalent, irreducible) pseudo Fock representations induced by $P^{\prime}$. 
We shall study the action of $\mathcal{I}_{P}^{0}(\mathcal{K}, \Gamma)$ on $\mathcal{I}_{P}(\mathcal{K}, \Gamma)$ in Section 4.3. Then the orbits are the sets $\mathcal{I}_{P}^{2 m}(\mathcal{K}, \Gamma), m \leq \infty$, and each orbit contains representatives $W$ with $\left[P, W W^{*}\right]=0$.

\section{Construction of Implementing Isometries}

Our construction of implementers follows the lines of Ruijsenaars' approach in [8] which is to our knowledge the most complete treatment of the implementation of Bogoliubov automorphisms. Another advantage of [8] for our purposes is the (implicit) use of Araki's selfdual CAR algebra formalism.

Let us first introduce some notation, followed by simple observations. Throughout this section $P_{1}$ is a fixed basis projection of $(\mathcal{K}, \Gamma)$ and $P_{2}:=\mathbf{1}-P_{1}=\overline{P_{1}}$. The components of an operator $A$ on $\mathcal{K}$ are denoted by

$$
A_{m n}:=P_{m} A P_{n}, \quad m, n=1,2
$$

and are regarded as operators from $\mathcal{K}_{n}:=P_{n}(\mathcal{K})$ to $\mathcal{K}_{m}$. Thus ker $A_{m n},\left(\operatorname{ker} A_{m n}\right)^{\perp},\left(\operatorname{ran} A_{n m}\right)^{\perp}$ etc. are viewed as subspaces of $\mathcal{K}_{n}$, and we have

$$
A_{m n}{ }^{*}=A^{*}{ }_{n m}, \quad \overline{A_{11}}=\bar{A}_{22} \quad \text { etc. }
$$

We also use matrix notation $A=\left(\begin{array}{ll}A_{11} & A_{12} \\ A_{21} & A_{22}\end{array}\right)$ w.r.t. to the decomposition $\mathcal{K}=\mathcal{K}_{1} \oplus \mathcal{K}_{2}$.

Let $V \in \mathcal{I}_{P_{1}}(\mathcal{K}, \Gamma)$ be a fixed Bogoliubov operator, with $\varrho_{V}$ implementable in the Fock representation $\pi_{P_{1}}$. The relation $V^{*} V=\mathbf{1}$ reads in components

$$
\begin{aligned}
& V_{11}{ }^{*} V_{11}+V_{21}{ }^{*} V_{21}=P_{1}, \\
& V_{22}{ }^{*} V_{22}+V_{12}{ }^{*} V_{12}=P_{2}, \\
& V_{11}{ }^{*} V_{12}+V_{21}{ }^{*} V_{22}=0, \\
& V_{22}{ }^{*} V_{21}+V_{12}{ }^{*} V_{11}=0,
\end{aligned}
$$

whereas $V=\bar{V}$ gives

$$
V_{22}=\overline{V_{11}}, \quad V_{21}=\overline{V_{12}} .
$$

Since $V_{12}$ is a Hilbert-Schmidt operator by Theorem 3.3, $V_{22}{ }^{*} V_{22}$ is Fredholm (with vanishing index) by (20). This means in particular

$$
\operatorname{dim} \operatorname{ker} V_{22}=\operatorname{dim} \operatorname{ker} V_{22}{ }^{*} V_{22}<\infty .
$$

Note that $\left.V_{12}\right|_{\operatorname{ker} V_{22}}$ is isometric and, by (21),

$$
V_{12}\left(\operatorname{ker} V_{22}\right) \subset \operatorname{ker} V_{11}{ }^{*} \text {. }
$$

As mentioned at the end of Section 3.1, $V_{11}{ }^{*}$ is semi-Fredholm with ind $V_{11}{ }^{*}=\frac{1}{2}$ ind $V^{*}$. By the above and by $\operatorname{ker} V_{11}=\Gamma \operatorname{ker} V_{22}$, we have

$$
\text { ind } V_{11}{ }^{*}=\operatorname{dim}\left(\operatorname{ker} V_{11}{ }^{*} \ominus V_{12}\left(\operatorname{ker} V_{22}\right)\right) \text {. }
$$

In the following, we are going to describe some operators by integral kernels. Thus we assume in this section (without loss of generality)

$$
\mathcal{K}_{1}=L^{2}\left(\mathbb{R}^{d}\right)
$$




\subsection{Unbounded Bilinear Hamiltonians and Ruijsenaars' Operator $\Lambda$}

Bounded bilinear Hamiltonians have been introduced by Araki [20] as infinitesimal generators of oneparameter groups of inner Bogoliubov automorphisms. More specifically, one may assign to a finite rank operator $H=\sum_{j} k_{j}\left\langle k_{j}^{\prime},.\right\rangle$ on $\mathcal{K}$ the bilinear Hamiltonian

$$
b(H):=\sum_{j} B\left(k_{j}\right) B\left(k_{j}^{\prime}\right)^{*}
$$

and extend $b$ to a linear map from $\mathfrak{J}_{1}(\mathcal{K})$ to $\mathcal{C}(\mathcal{K}, \Gamma)$ by continuity (relative to trace norm on $\mathfrak{J}_{1}(\mathcal{K})$ and $\mathrm{C}^{*}$-norm on $\mathcal{C}(\mathcal{K}, \Gamma)$ ). If $H \in \mathfrak{J}_{1}(\mathcal{K})$ satisfies $H^{*}=-H$ and $\bar{H}=H$, then $b(H) / 2$ is the generator of the one-parameter group $\left(\alpha_{e^{t H}}\right)_{t \in \mathbb{R}}$ :

$$
\alpha_{e^{t H}}=\operatorname{Ad}(\exp (t b(H) / 2)) .
$$

Further properties of $b$ are summarized in 12, 15.

Since elements $B(k)$ with $k \in \mathcal{K}_{1}$ correspond to creation operators in the Fock representation $\pi_{P_{1}}$, we may write

$$
\pi_{P_{1}}(b(H))=H_{12} a^{*} a^{*}+H_{11} a^{*} a+H_{22} a a^{*}+H_{21} a a
$$

where the terms on the right are defined by $H_{12} a^{*} a^{*}:=\pi_{P_{1}}\left(b\left(H_{12}\right)\right)$ etc. Introducing Wick ordering by $: a(f) a(g)^{*}:=-a(g)^{*} a(f)$, we get $: H_{22} a a^{*}:=-{\overline{H_{22}}}^{*} a^{*} a=H_{22} a a^{*}-\left(\operatorname{tr} H_{22}\right) \mathbf{1}$ and

$$
: \pi_{P_{1}}(b(H)):=H_{12} a^{*} a^{*}+\left(H_{11}-\overline{H_{22}{ }^{*}}\right) a^{*} a+H_{21} a a .
$$

According to 8, 34], one may define such Wick ordered expressions for bounded $H$ as follows. Let $\mathcal{S} \subset \mathcal{F}_{a}\left(\mathcal{K}_{1}\right)$ be the dense subspace consisting of finite particle vectors $\phi$ with $n$-particle wave functions $\phi^{(n)}$ in the Schwartz space $\mathcal{S}\left(\mathbb{R}^{d n}\right)$. For $p \in \mathbb{R}^{d}$, the unsmeared annihilation operator $a(p)$ with (invariant) domain $\mathcal{S}$ is defined by

$$
(a(p) \phi)^{(n)}\left(p_{1}, \ldots, p_{n}\right):=\sqrt{n+1} \phi^{(n+1)}\left(p, p_{1}, \ldots, p_{n}\right) .
$$

Since $a(p)$ is not closable, one defines $a(p)^{*}$ as the quadratic form adjoint of $a(p)$ on $\mathcal{S} \times \mathcal{S}$. Then Wick ordered monomials $a\left(q_{m}\right)^{*} \cdots a\left(q_{1}\right)^{*} a\left(p_{1}\right) \cdots a\left(p_{n}\right)$ are well-defined quadratic forms on $\mathcal{S} \times \mathcal{S}$, and for $\phi, \phi^{\prime} \in \mathcal{S}$,

$$
\left\langle\phi, a\left(q_{m}\right)^{*} \cdots a\left(q_{1}\right)^{*} a\left(p_{1}\right) \cdots a\left(p_{n}\right) \phi^{\prime}\right\rangle=\left\langle a\left(q_{1}\right) \cdots a\left(q_{m}\right) \phi, a\left(p_{1}\right) \cdots a\left(p_{n}\right) \phi^{\prime}\right\rangle
$$

is a function in $\mathcal{S}\left(\mathbb{R}^{d(m+n)}\right)$ to which tempered distributions may be applied. For example, one has in the quadratic form sense

$$
a(f)=\int \overline{f(p)} a(p) d p, \quad a(f)^{*}=\int f(p) a(p)^{*} d p, \quad f \in \mathcal{K}_{1} .
$$

Now let $H$ be a bounded operator on $\mathcal{K}$. By the nuclear theorem of Schwartz, there exist tempered distributions $H_{m n}(p, q), m, n=1,2$, given by

$$
\begin{aligned}
\left\langle f, H_{11} g\right\rangle & =\int \overline{f(p)} H_{11}(p, q) g(q) d p d q \\
\left\langle f, H_{12} \Gamma g\right\rangle & =\int \overline{f(p)} H_{12}(p, q) \overline{g(q)} d p d q \\
\left\langle\Gamma f, H_{21} g\right\rangle & =\int f(p) H_{21}(p, q) \underline{g(q)} d p d q, \\
\left\langle\Gamma f, H_{22} \Gamma g\right\rangle & =\int f(p) H_{22}(p, q) g(q) d p d q, \quad f, g \in \mathcal{S}\left(\mathbb{R}^{d}\right) \subset \mathcal{K}_{1} .
\end{aligned}
$$


Hence we may define the following quadratic forms on $\mathcal{S} \times \mathcal{S}$

$$
\begin{aligned}
H_{12} a^{*} a^{*} & :=\int H_{12}(p, q) a(p)^{*} a(q)^{*} d p d q \\
H_{11} a^{*} a & :=\int H_{11}(p, q) a(p)^{*} a(q) d p d q \\
: H_{22} a a^{*}: & :=-H_{22}{ }^{*} a^{*} a \\
& =-\int H_{22}(q, p) a(p)^{*} a(q) d p d q \\
H_{21} a a & :=\int H_{21}(p, q) a(p) a(q) d p d q .
\end{aligned}
$$

The (Wick ordered, unbounded) bilinear Hamiltonian induced by $H$ is then defined in analogy to (26) as

$$
: b(H)::=H_{12} a^{*} a^{*}+\left(H_{11}-\overline{H_{22}{ }^{*}}\right) a^{*} a+H_{21} a a
$$

it is linear in $H$. We define its Wick ordered powers as

$$
: b(H)^{l}::=l ! \sum_{\substack{l_{1}, l_{2}, l_{3}=0 \\ l_{1}+l_{2}+l_{3}=l}}^{l} \frac{1}{l_{1} ! l_{2} ! l_{3} !}\left(H_{12}\right)^{l_{1}}\left(H_{11}-\overline{H_{22}{ }^{*}}\right)^{l_{2}}\left(H_{21}\right)^{l_{3}} a^{* 2 l_{1}+l_{2}} a^{l_{2}+2 l_{3}}
$$

where the terms on the right hand side are quadratic forms on $\mathcal{S} \times \mathcal{S}(\mathrm{cf}$. [] $)$

$$
\begin{aligned}
& \left(H_{12}\right)^{l_{1}}\left(H_{11}-\overline{H_{22}{ }^{*}}\right)^{l_{2}}\left(H_{21}\right)^{l_{3}} a^{* 2 l_{1}+l_{2}} a^{l_{2}+2 l_{3}}:= \\
& \quad \int H_{12}\left(p_{1}, q_{1}\right) \cdots H_{12}\left(p_{l_{1}}, q_{l_{1}}\right)\left(H_{11}\left(p_{1}^{\prime}, q_{1}^{\prime}\right)-H_{22}\left(q_{1}^{\prime}, p_{1}^{\prime}\right)\right) \cdots\left(H_{11}\left(p_{l_{2}}^{\prime}, q_{l_{2}}^{\prime}\right)-H_{22}\left(q_{l_{2}}^{\prime}, p_{l_{2}}^{\prime}\right)\right) \cdot \\
& \quad \cdot H_{21}\left(p_{1}^{\prime \prime}, q_{1}^{\prime \prime}\right) \cdots H_{21}\left(p_{l_{3}}^{\prime \prime}, q_{l_{3}}^{\prime \prime}\right) a\left(p_{1}\right)^{*} \cdots a\left(p_{l_{1}}\right)^{*} a\left(q_{l_{1}}\right)^{*} \cdots a\left(q_{1}\right)^{*} a\left(p_{1}^{\prime}\right)^{*} \cdots a\left(p_{l_{2}}^{\prime}\right)^{*} a\left(q_{l_{2}}^{\prime}\right) \cdots a\left(q_{1}^{\prime}\right) \\
& \quad \cdot a\left(p_{1}^{\prime \prime}\right) \cdots a\left(p_{l_{3}}^{\prime \prime}\right) a\left(q_{l_{3}}^{\prime \prime}\right) \cdots a\left(q_{1}^{\prime \prime}\right) d p_{1} d q_{1} \ldots d p_{l_{1}} d q_{l_{1}} d p_{1}^{\prime} d q_{1}^{\prime} \ldots d p_{l_{2}}^{\prime} d q_{l_{2}}^{\prime} d p_{1}^{\prime \prime} d q_{1}^{\prime \prime} \ldots d p_{l_{3}}^{\prime \prime} d q_{l_{3}}^{\prime \prime} .
\end{aligned}
$$

Finally, we define the Wick ordered exponential

$$
: \exp (b(H) / 2)::=\sum_{l=0}^{\infty} \frac{1}{l ! 2^{l}}: b(H)^{l}:
$$

which is also a well-defined quadratic form on $\mathcal{S} \times \mathcal{S}$ since the sum in (31) is finite when applied to vectors $\phi, \phi^{\prime} \in \mathcal{S}$.

How do we have to choose $H$ in order to relate these quadratic forms to implementers for $\varrho_{V}$ ? Let us first remark that we may restrict attention to antisymmetric $H$, i.e. to operators fulfilling

$$
H=-\overline{H^{*}}
$$

(in components: $H_{11}=-\overline{{H_{22}}^{*}}, H_{12}=-\overline{H_{12}{ }^{*}}, H_{21}=-\overline{H_{21}{ }^{*}}$ ). Indeed, we have $H=H^{+}+H^{-}$with $H^{ \pm}:=\frac{1}{2}\left(H \pm \overline{H^{*}}\right)= \pm \overline{\left(H^{ \pm}\right)^{*}}$, and we claim that

$$
: b(H):=: b\left(H^{-}\right):
$$

or equivalently $: b\left(H^{+}\right):=0$. From $\overline{\left(H_{22}^{+}\right)^{*}}=H_{11}^{+}$we infer $: b\left(H^{+}\right):=H_{12}^{+} a^{*} a^{*}+H_{21}^{+} a a$. It follows from $H_{12}^{+}=\overline{\left(H_{12}^{+}\right)^{*}}$ and $(27)$ that $H_{12}(p, q)=H_{12}(q, p)$. But by virtue of the CAR we have $H_{12}^{+} a^{*} a^{*}=$ $\int H_{12}(p, q) a(p)^{*} a(q)^{*} d p d q=-\int H_{12}(q, p) a(q)^{*} a(p)^{*} d p d q=-H_{12}^{+} a^{*} a^{*}$, hence $H_{12}^{+} a^{*} a^{*}=0$. A similar argument shows $H_{21}^{+} a a=0$ which proves the assertion.

So let $H$ be antisymmetric in the above sense. Of course, we would like to deal with well-defined operators instead of quadratic forms. By a result of Ruijsenaars [8], $: \exp (b(H) / 2)$ : is the quadratic 
form of a densely defined operator with domain $\mathcal{D}:=\pi_{P_{1}}\left(\mathcal{C}_{0}(\mathcal{K}, \Gamma)\right) \Omega_{P_{1}}$, the subspace of algebraic tensors in $\mathcal{F}_{a}\left(\mathcal{K}_{1}\right)$, provided that $H_{12}$ is Hilbert-Schmidt. In this case, the series (31) converges strongly on $\mathcal{D}$, : $\exp (b(H) / 2)$ : (viewed as an operator) maps $\mathcal{D}$ into the dense subspace of $C^{\infty}$-vectors for the number operator, and

$$
\left\|: \exp (b(H) / 2): \Omega_{P_{1}}\right\|=\left(\operatorname{det}\left(P_{1}+H_{12} H_{12}{ }^{*}\right)\right)^{1 / 4} .
$$

The operators $H$ of interest are now selected by intertwining properties (cf. (14)) of : $\exp (b(H) / 2)$ : Let $a_{V}(f)=a\left(V_{11} f\right)+a\left(V_{12} \Gamma f\right)^{*}$ denote the transformed annihilation operator as in (3). We are looking for operators $H$ fulfilling

$$
\begin{aligned}
: \exp (b(H) / 2): a(f)^{*} & =a_{V}(f)^{*}: \exp (b(H) / 2):, \quad f \in \mathcal{K}_{1} \\
: \exp (b(H) / 2): a(g) & =a_{V}(g): \exp (b(H) / 2):, \quad g \in\left(\operatorname{ker} V_{11}\right)^{\perp}
\end{aligned}
$$

on $\mathcal{D}$. Since $a_{V}(g)$ is a creation operator for $g \in \operatorname{ker} V_{11}$, (35) cannot hold for such $g$ unless $g=0$ (the l.h.s. of (35) vanishes on $\Omega_{P_{1}}$, but the r.h.s. does not, cf. the proof of Lemma 4.2). We impose an additional relation for vectors in ker $V_{11}$ which will prove to be "correct":

$$
: \exp (b(H) / 2): a(h)^{*}=0, \quad h \in \operatorname{ker} V_{11} .
$$

To solve (34)-(36), we have to compute commutation relations of $: \exp (b(H) / 2)$ : with creation and annihilation operators.

Lemma 4.1 Let $H \in \mathfrak{B}(\mathcal{K})$ be antisymmetric in the sense of (39) with $H_{12}$ Hilbert-Schmidt. For $f, g \in \mathcal{K}_{1}$, the following relations hold on $\mathcal{D}$

$$
\begin{aligned}
{\left[: \exp (b(H) / 2):, a(f)^{*}\right] } & =a\left(H_{11} f\right)^{*}: \exp (b(H) / 2):+: \exp (b(H) / 2): a\left(\Gamma H_{21} f\right), \\
{[: \exp (b(H) / 2):, a(g)] } & =a\left(H_{12} \Gamma g\right)^{*}: \exp (b(H) / 2):+: \exp (b(H) / 2): a\left(\overline{H_{22}} g\right) .
\end{aligned}
$$

Proof. Let us first compute commutation relations for Wick monomials of the form (cf. (30))

$$
H_{l_{1}, l_{2}, l_{3}}:=\left(H_{12}\right)^{l_{1}}\left(H_{11}\right)^{l_{2}}\left(H_{21}\right)^{l_{3}} a^{* 2 l_{1}+l_{2}} a^{l_{2}+2 l_{3}} .
$$

Using the formal CAR, we get

$$
\begin{aligned}
& a\left(q_{l}\right) \cdots a\left(q_{1}\right) a(p)^{*}=(-1)^{l} a(p)^{*} a\left(q_{l}\right) \cdots a\left(q_{1}\right)+\sum_{j=1}^{l}(-1)^{j-1} \delta\left(q_{j}-p\right) a\left(q_{l}\right) \cdots \widetilde{a\left(q_{j}\right)} \cdots a\left(q_{1}\right), \\
& (-1)^{l} a\left(p_{1}\right) \cdots a\left(p_{l}\right) a(p)^{*}=a(p)^{*} a\left(p_{1}\right) \cdots a\left(p_{l}\right)+\sum_{j=1}^{l}(-1)^{j} \delta\left(p_{j}-p\right) a\left(p_{1}\right) \cdots \widetilde{a\left(p_{j}\right)} \cdots a\left(p_{l}\right),
\end{aligned}
$$

where the factors under the symbol " $"$ are to be omitted. In the following computation, we use in addition

$$
\begin{gathered}
\int a(p) H_{21}(p, q) f(q) d p d q=a\left(\Gamma H_{21} f\right), \\
\int f(p) H_{21}(p, q) a(q) d p d q=a\left(H_{21}{ }^{*} \Gamma f\right)=-a\left(\Gamma H_{21} f\right), \\
\int a(p)^{*} H_{11}(p, q) f(q) d p d q=a\left(H_{11} f\right)^{*} . \\
H_{l_{1}, l_{2}, l_{3}} a(f)^{*}=\int H_{12}\left(p_{1}, q_{1}\right) \cdots H_{12}\left(p_{l_{1}}, q_{l_{1}}\right) H_{11}\left(p_{1}^{\prime}, q_{1}^{\prime}\right) \cdots H_{11}\left(p_{l_{2}}^{\prime}, q_{l_{2}}^{\prime}\right) H_{21}\left(p_{1}^{\prime \prime}, q_{1}^{\prime \prime}\right) \cdots H_{21}\left(p_{l_{3}}^{\prime \prime}, q_{l_{3}}^{\prime \prime}\right) . \\
\cdot f(p) a\left(p_{1}\right)^{*} \cdots a\left(p_{l_{1}}\right)^{*} a\left(q_{l_{1}}\right)^{*} \cdots a\left(q_{1}\right)^{*} a\left(p_{1}^{\prime}\right)^{*} \cdots a\left(p_{l_{2}}^{\prime}\right)^{*} a\left(q_{l_{2}}^{\prime}\right) \cdots a\left(q_{1}^{\prime}\right) a\left(p_{1}^{\prime \prime}\right) \cdots a\left(p_{l_{3}}^{\prime \prime}\right) \cdot \\
\cdot a\left(q_{l_{3}}^{\prime \prime}\right) \cdots a\left(q_{1}^{\prime \prime}\right) a(p)^{*} d p_{1} d q_{1} \ldots d p_{l_{1}} d q_{l_{1}} d p_{1}^{\prime} d q_{1}^{\prime} \ldots d p_{l_{2}}^{\prime} d q_{l_{2}}^{\prime} d p_{1}^{\prime \prime} d q_{1}^{\prime \prime} \ldots d p_{l_{3}}^{\prime \prime} d q_{l_{3}}^{\prime \prime} d p \\
=(-1)^{l_{3}} \int H_{12}\left(p_{1}, q_{1}\right) \cdots H_{21}\left(p_{l_{3}}^{\prime \prime}, q_{l_{3}}^{\prime \prime}\right) f(p) a\left(p_{1}\right)^{*} \cdots a\left(q_{1}^{\prime}\right) a\left(p_{1}^{\prime \prime}\right) \cdots a\left(p_{l_{3}}^{\prime \prime}\right) a(p)^{*} a\left(q_{l_{3}}^{\prime \prime}\right) \cdots a\left(q_{1}^{\prime \prime}\right) d p_{1} \ldots d p
\end{gathered}
$$




$$
\begin{aligned}
& +\sum_{j=1}^{l_{3}}(-1)^{j-1} \int H_{12}\left(p_{1}, q_{1}\right) \cdots H_{21}\left(p_{j}^{\prime \prime}, q_{j}^{\prime \prime}\right) \cdots H_{21}\left(p_{l_{3}}^{\prime \prime}, q_{l_{3}}^{\prime \prime}\right) \delta\left(q_{j}^{\prime \prime}-p\right) f(p) \cdot \\
& \cdot a\left(p_{1}\right)^{*} \cdots a\left(q_{1}^{\prime}\right) a\left(p_{1}^{\prime \prime}\right) \cdots \widetilde{a\left(p_{j}^{\prime \prime}\right)} \cdots a\left(p_{l_{3}}^{\prime \prime}\right) a\left(q_{l_{3}}^{\prime \prime}\right) \cdots \widetilde{a\left(q_{j}^{\prime \prime}\right)} \cdots a\left(q_{1}^{\prime \prime}\right)(-1)^{l_{3}-j+l_{3}-1} a\left(p_{j}^{\prime \prime}\right) d p_{1} \ldots d p \\
= & \int H_{12}\left(p_{1}, q_{1}\right) \cdots H_{21}\left(p_{l_{3}}^{\prime \prime}, q_{l_{3}}^{\prime \prime}\right) f(p) a\left(p_{1}\right)^{*} \cdots a\left(p_{l_{2}}^{\prime}\right)^{*} a\left(q_{l_{2}}^{\prime}\right) \cdots a\left(q_{1}^{\prime}\right) a(p)^{*} a\left(p_{1}^{\prime \prime}\right) \cdots a\left(q_{1}^{\prime \prime}\right) d p_{1} \ldots d p \\
& +\sum_{j=1}^{l_{3}}(-1)^{j} \int H_{12}\left(p_{1}, q_{1}\right) \cdots H_{21}\left(p_{j}^{\prime \prime}, q_{j}^{\prime \prime}\right) \cdots H_{21}\left(p_{l_{3}}^{\prime \prime}, q_{l_{3}}^{\prime \prime}\right) \delta\left(p_{j}^{\prime \prime}-p\right) f(p) a\left(p_{1}\right)^{*} \cdots a\left(q_{1}^{\prime}\right) a\left(p_{1}^{\prime \prime}\right) \cdots \\
& \cdots \widetilde{a\left(p_{j}^{\prime \prime}\right)} \cdots a\left(p_{l_{3}}^{\prime \prime}\right) a\left(q_{l_{3}}^{\prime \prime}\right) \cdots \widetilde{a\left(q_{j}^{\prime \prime}\right)} \cdots a\left(q_{1}^{\prime \prime}\right)(-1)^{j-1} a\left(q_{j}^{\prime \prime}\right) d p_{1} \ldots d p+l_{3} H_{l_{1}, l_{2}, l_{3}-1} a\left(\Gamma H_{21} f\right) \\
= & (-1)^{l_{2}} \int H_{12}\left(p_{1}, q_{1}\right) \cdots H_{21}\left(p_{l_{3}}^{\prime \prime}, q_{l_{3}}^{\prime \prime}\right) f(p) a\left(p_{1}\right)^{*} \cdots a\left(p_{l_{2}}^{\prime}\right)^{*} a(p)^{*} a\left(q_{l_{2}}^{\prime}\right) \cdots a\left(q_{1}^{\prime \prime}\right) d p_{1} \ldots d p \\
& +\sum_{j=1}^{l_{2}}(-1)^{j-1} \int H_{12}\left(p_{1}, q_{1}\right) \cdots H_{11}\left(p_{j}^{\prime}, q_{j}^{\prime}\right) \cdots H_{21}\left(p_{l_{3}}^{\prime \prime}, q_{l_{3}}^{\prime \prime}\right) \delta\left(q_{j}^{\prime}-p\right) f(p)(-1)^{j-1} a\left(p_{j}^{\prime}\right)^{*} . \\
& \cdot a\left(p_{1}\right)^{*} \cdots a\left(q_{1}\right)^{*} a\left(p_{1}^{\prime}\right)^{*} \cdots \widetilde{a\left(p_{j}^{\prime}\right)^{*}} \cdots a\left(p_{l_{2}}^{\prime}\right)^{*} a\left(q_{l_{2}}^{\prime}\right) \cdots \widetilde{a\left(q_{j}^{\prime}\right)} \cdots a\left(q_{1}^{\prime}\right) a\left(p_{1}^{\prime \prime}\right) \cdots a\left(q_{1}^{\prime \prime}\right) d p_{1} \ldots d p \\
& +2 l_{3} H_{l_{1}, l_{2}, l_{3}-1} a\left(\Gamma H_{21} f\right) \\
= & a(f)^{*} H_{l_{1}, l_{2}, l_{3}}+l_{2} a\left(H_{11} f\right)^{*} H_{l_{1}, l_{2}-1, l_{3}}+2 l_{3} H_{l_{1}, l_{2}, l_{3}-1} a\left(\Gamma H_{21} f\right) .
\end{aligned}
$$

Hence we have on $\mathcal{D}: \quad\left[H_{l_{1}, l_{2}, l_{3}}, a(f)^{*}\right]=l_{2} a\left(H_{11} f\right)^{*} H_{l_{1}, l_{2}-1, l_{3}}+2 l_{3} H_{l_{1}, l_{2}, l_{3}-1} a\left(\Gamma H_{21} f\right)$.

Taking into account

$$
\begin{aligned}
& (-1)^{l} a\left(p_{1}\right)^{*} \cdots a\left(p_{l}\right)^{*} a(p)=a(p) a\left(p_{1}\right)^{*} \cdots a\left(p_{l}\right)^{*}+\sum_{j=1}^{l}(-1)^{j} \delta\left(p-p_{j}\right) a\left(p_{1}\right)^{*} \cdots \widetilde{a\left(p_{j}\right)^{*}} \cdots a\left(p_{l}\right)^{*}, \\
& a\left(q_{l}\right)^{*} \cdots a\left(q_{1}\right)^{*} a(p)=(-1)^{l} a(p) a\left(q_{l}\right)^{*} \cdots a\left(q_{1}\right)^{*}+\sum_{j=1}^{l}(-1)^{j-1} \delta\left(p-q_{j}\right) a\left(q_{l}\right)^{*} \cdots \widetilde{a\left(q_{j}\right)^{*}} \cdots a\left(q_{1}\right)^{*}
\end{aligned}
$$

and

$$
\begin{aligned}
& \int \overline{g(p)} H_{11}(p, q) a(q) d p d q=a\left(H_{11}{ }^{*} g\right) \quad=-a\left(\overline{H_{22}} g\right), \\
& \int a(p)^{*} H_{12}(p, q) \overline{g(q)} d p d q=a\left(H_{12} \Gamma g\right)^{*}, \\
& \int \overline{g(p)} H_{12}(p, q) a(q)^{*} d p d q=a\left(\Gamma H_{12}{ }^{*} g\right)^{*}=-a\left(H_{12} \Gamma g\right)^{*} \text {, }
\end{aligned}
$$

we find in a similar way: $\left[H_{l_{1}, l_{2}, l_{3}}, a(g)\right]=2 l_{1} a\left(H_{12} \Gamma g\right)^{*} H_{l_{1}-1, l_{2}, l_{3}}+l_{2} H_{l_{1}, l_{2}-1, l_{3}} a\left(\overline{H_{22}} g\right)$.

Combination of these commutation relations with (29)-(32) now yields

$$
\begin{aligned}
{\left[: \exp (b(H) / 2):, a(f)^{*}\right]=} & \sum_{l=0}^{\infty} 2^{-l} \sum_{l_{1}+l_{2}+l_{3}=l} \frac{2^{l_{2}}}{l_{1} ! l_{2} ! l_{3} !}\left[H_{l_{1}, l_{2}, l_{3}}, a(f)^{*}\right] \\
= & a\left(H_{11} f\right)^{*} \sum_{l=1}^{\infty} 2^{-(l-1)} \sum_{l_{1}+l_{2}+l_{3}=l} \frac{2^{l_{2}-1}}{l_{1} !\left(l_{2}-1\right) ! l_{3} !} H_{l_{1}, l_{2}-1, l_{3}} \\
& +\sum_{l=1}^{\infty} 2^{-(l-1)} \sum_{l_{1}+l_{2}+l_{3}=l} \frac{2^{l_{2}}}{l_{1} ! l_{2} !\left(l_{3}-1\right) !} H_{l_{1}, l_{2}, l_{3}-1} a\left(\Gamma H_{21} f\right) \\
= & a\left(H_{11} f\right)^{*}: \exp (b(H) / 2):+: \exp (b(H) / 2): a\left(\Gamma H_{21} f\right), \\
{[: \exp (b(H) / 2):, a(g)]=} & \sum_{l=0}^{\infty} 2^{-l} \sum_{l_{1}+l_{2}+l_{3}=l} \frac{2^{l_{2}}}{l_{1} ! l_{2} ! l_{3} !}\left[H_{\left.l_{1}, l_{2}, l_{3}, a(g)\right]}\right.
\end{aligned}
$$




$$
\begin{aligned}
= & a\left(H_{12} \Gamma g\right)^{*} \sum_{l=1}^{\infty} 2^{-(l-1)} \sum_{l_{1}+l_{2}+l_{3}=l} \frac{2^{l_{2}}}{\left(l_{1}-1\right) ! l_{2} ! l_{3} !} H_{l_{1}-1, l_{2}, l_{3}} \\
& +\sum_{l=1}^{\infty} 2^{-(l-1)} \sum_{l_{1}+l_{2}+l_{3}=l} \frac{2^{l_{2}-1}}{l_{1} !\left(l_{2}-1\right) ! l_{3} !} H_{l_{1}, l_{2}-1, l_{3}} a\left(\overline{H_{22}} g\right) \\
= & a\left(H_{12} \Gamma g\right)^{*}: \exp (b(H) / 2):+\exp (b(H) / 2): a\left(\overline{H_{22}} g\right) .
\end{aligned}
$$

We next introduce the associate [8] $\Lambda(V)$ of $V$ as a special solution of (34)-(36) f]. Since the ranges of the semi-Fredholm operators $V_{11}$ and $V_{11}{ }^{*}$ are closed, the bounded bijection $\left.V_{11}\right|_{\mathrm{ran} V_{11}}$ from $\operatorname{ran} V_{11}{ }^{*}=$ $\left(\operatorname{ker} V_{11}\right)^{\perp}$ onto ran $V_{11}$ has a bounded inverse. Let $V_{11}{ }^{-1} \in \mathfrak{B}\left(\mathcal{K}_{1}\right)$ equal this inverse on ran $V_{11}$ and equal zero on $\operatorname{ker} V_{11}{ }^{*}$. We then have

$$
\begin{array}{ll}
\operatorname{ran} V_{11}{ }^{-1}=\operatorname{ran} V_{11}{ }^{*}, & \operatorname{ker} V_{11}{ }^{-1}=\operatorname{ker} V_{11}{ }^{*}, \\
V_{11} V_{11}{ }^{-1}=P_{\operatorname{ran} V_{11}}, & V_{11}{ }^{-1} V_{11}=P_{\operatorname{ran} V_{11}}{ }^{*}
\end{array}
$$

where $P_{\mathcal{H}}$ denotes the orthogonal projection onto a closed subspace $\mathcal{H} \subset \mathcal{K}$. Of course, the analogous relations hold true for $V_{22}^{-1}=\Gamma V_{11}^{-1} \Gamma$. As a generalization of Ruijsenaars' definition in [8], we now set

$$
\begin{aligned}
& \Lambda(V)_{12}:=V_{12} V_{22}{ }^{-1}-V_{11}{ }^{-1 *} V_{21}{ }^{*} P_{\text {ker } V_{22}{ }^{*}} \\
& \Lambda(V)_{11}:=V_{11}{ }^{-1 *}-P_{1}-P_{\text {ker }} V_{11}{ }_{12} V_{22} V_{22} V_{21} \\
& \Lambda(V)_{22}:=P_{2}-V_{22}{ }^{-1}+V_{12}{ }^{*} V_{11}{ }^{-1 *} V_{21}{ }^{*} P_{\text {ker }} V_{22}{ }^{*} \\
& \Lambda(V)_{21}:=\left(V_{22}{ }^{-1}-V_{12}{ }^{*} V_{11}{ }^{-1 *} V_{21}{ }^{*} P_{\text {ker } V_{22}}{ }^{*}\right) V_{21}
\end{aligned}
$$

Lemma 4.2 The antisymmetric solutions $H$ of (34)-(30) with $H_{12}$ Hilbert-Schmidt are precisely the operators of the form

$$
H=\Lambda(V)+\left(\begin{array}{cc}
-h_{12} V_{21} & h_{12} \\
V_{12}{ }^{*} h_{12} V_{21} & -V_{12}{ }^{*} h_{12}
\end{array}\right)
$$

where $h_{12}$ is an antisymmetric Hilbert-Schmidt operator from $\mathcal{K}_{2}$ to $\mathcal{K}_{1}$ with

$$
\left(\operatorname{ker} h_{12}\right)^{\perp} \subset \operatorname{ker} V_{22}{ }^{*} \ominus V_{21}\left(\operatorname{ker} V_{11}\right), \quad \operatorname{ran} h_{12} \subset \operatorname{ker} V_{11}{ }^{*} \ominus V_{12}\left(\operatorname{ker} V_{22}\right) .
$$

The space spanned by such operators $h_{12}$ has dimension $\left(m^{2}-m\right) / 2, \quad m:=\operatorname{ind} V_{11}{ }^{*}=\frac{1}{2}$ ind $V^{*}$.

Proof. We first note that a Wick ordered expression $a(f)^{*}: \exp (b(H) / 2):+: \exp (b(H) / 2): a(g)$ vanishes if and only if $f=g=0$. In fact, application to the vacuum gives $a(f)^{*} \exp \left(\frac{1}{2} H_{12} a^{*} a^{*}\right) \Omega_{P_{1}}$ which equals zero if and only if $f=0$ (to see this, look for instance at the one-particle component). Similarly, $(: \exp (b(H) / 2): a(g)) a(g)^{*} \Omega_{P_{1}}=\|g\|^{2} \exp \left(\frac{1}{2} H_{12} a^{*} a^{*}\right) \Omega_{P_{1}}$ vanishes if and only if $g=0$.

Hence we get all solutions of (34)-(36) if we write these equations in Wick ordered form and then compare term by term. We have by Lemma 4.1

$$
\begin{aligned}
: \exp (b(H) / 2): a(f)^{*} & =a\left(\left(P_{1}+H_{11}\right) f\right)^{*}: \exp (b(H) / 2):+\exp (b(H) / 2): a\left(\Gamma H_{21} f\right), \\
a_{V}(f)^{*}: \exp (b(H) / 2): & =a\left(\left(V_{11}-H_{12} V_{21}\right) f\right)^{*}: \exp (b(H) / 2):+: \exp (b(H) / 2): a\left(\Gamma\left(P_{2}-H_{22}\right) V_{21} f\right), \\
a_{V}(g): \exp (b(H) / 2): & =a\left(\left(V_{12}-H_{12} V_{22}\right) \Gamma g\right)^{*}: \exp (b(H) / 2):+\exp (b(H) / 2): a\left(\left(P_{1}-\overline{H_{22}}\right) V_{11} g\right) .
\end{aligned}
$$

\footnotetext{
${ }^{f}$ The operators $H_{12}$ described below may equivalently be characterized as follows. According to Lemma 4.6, each antisymmetric Hilbert-Schmidt operator $T$ from $\mathcal{K}_{1}$ to $\mathcal{K}_{2}$ induces a basis projection $P_{T}$. Then $V^{*} P_{T} V=W^{*} P_{1} W$ (see Lemma 4.8) holds if and only if $-T^{*}=H_{12}$ for some $H$ as in Lemma 4.2
} 
Thus (34) is equivalent to

$$
\begin{aligned}
\left(P_{1}+H_{11}-V_{11}+H_{12} V_{21}\right) f & =0, \\
\left(H_{21}+\left(H_{22}-P_{2}\right) V_{21}\right) f & =0, \quad f \in \mathcal{K}_{1}
\end{aligned}
$$

(35) is equivalent to

$$
\begin{aligned}
\left(H_{12} V_{22}-V_{12}\right) \Gamma g & =0, \\
\left(P_{1}+\left(\overline{H_{22}}-P_{1}\right) V_{11}\right) g & =0, \quad g \in \operatorname{ran} V_{11}{ }^{*}
\end{aligned}
$$

whereas (36) is equivalent to

$$
\begin{aligned}
\left(P_{1}+H_{11}\right) h & =0, \\
H_{21} h & =0, \quad h \in \operatorname{ker} V_{11} .
\end{aligned}
$$

Next we show that each antisymmetric Hilbert-Schmidt operator $H_{12}$ fulfilling (41) and $V_{21}\left(\operatorname{ker} V_{11}\right) \subset$ ker $H_{12}$ gives rise to a unique solution $H$ of (32) and (34)-(36). Given $H_{12}, H_{11}$ is fixed by (39) which in turn yields $H_{22}=-\overline{H_{11}{ }^{*}}$ by (32). $H_{21}$ is then determined by (40) which proves uniqueness of $H$. Explicitly, we have

$$
H_{11}=V_{11}-P_{1}-H_{12} V_{21}, \quad H_{22}=P_{2}-V_{22}{ }^{*}-V_{12}{ }^{*} H_{12}, \quad H_{21}=\left(V_{22}{ }^{*}+V_{12}{ }^{*} H_{12}\right) V_{21} .
$$

To see that $H$ indeed is a solution of (32) and (34)-(36), we have to check antisymmetry of $H_{21}$, (42) and (43) (the rest is clear by construction). By antisymmetry of $H_{12}$ and (22), we have

$$
H_{21}+\overline{H_{21}{ }^{*}}=\left(V_{22}{ }^{*}+V_{12}{ }^{*} H_{12}\right) V_{21}+V_{12}{ }^{*}\left(V_{11}-H_{12} V_{21}\right)=0,
$$

so $H_{21}$ is antisymmetric. By (19) and (41), we have for $g \in \operatorname{ran} V_{11}{ }^{*}$

$$
\left(P_{1}+\left(\overline{H_{22}}-P_{1}\right) V_{11}\right) g=\left(P_{1}-\left(V_{11}{ }^{*}+V_{21} * \overline{H_{12}}\right) V_{11}\right) g=V_{21}{ }^{*} \Gamma\left(V_{12}-H_{12} V_{22}\right) \Gamma g=0,
$$

so (42) holds. Using (22), we find for $h \in \operatorname{ker} V_{11}$

$$
\left(P_{1}+H_{11}\right) h=-H_{12} V_{21} h, \quad H_{21} h=V_{12}{ }^{*} H_{12} V_{21} h .
$$

Thus (43) is equivalent to $V_{21}\left(\operatorname{ker} V_{11}\right) \subset \operatorname{ker} H_{12}$ which holds by assumption, so $H$ solves (32) and (34)-(36).

Finally, we have to characterize the antisymmetric Hilbert-Schmidt operators $H_{12}$ fulfilling (41) and $V_{21}\left(\operatorname{ker} V_{11}\right) \subset \operatorname{ker} H_{12}$. Note that $\Lambda(V)_{12}$ is Hilbert-Schmidt since $V_{12}$ is, that $\Lambda(V)_{12}$ solves (41) and that

$$
\Lambda(V)_{12} V_{21} h=-V_{11}{ }^{-1 *} V_{21}{ }^{*} V_{21} h=-V_{11}{ }^{-1 *} h=0, \quad h \in \operatorname{ker} V_{11}=\operatorname{ker} V_{11}{ }^{-1 *}
$$

by (24), (37) and (19). $\Lambda(V)_{12}$ is also antisymmetric:

$$
\begin{aligned}
\Lambda(V)_{12}+\overline{\Lambda(V)_{12}{ }^{*}} & =V_{12} V_{22}{ }^{-1}-V_{11}{ }^{-1 *} V_{21}{ }^{*} P_{\text {ker } V_{22}{ }^{*}+V_{11}{ }^{-1 *} V_{21}{ }^{*}-P_{\text {ker } V_{11}}{ }^{*} V_{12} V_{22}{ }^{-1}} \\
& =P_{\operatorname{ran} V_{11}} V_{12} V_{22}{ }^{-1}+V_{11}{ }^{-1 *} V_{21}{ }^{*} P_{\operatorname{ran} V_{22}} \\
& =V_{11}{ }^{-1 *}\left(V_{11}{ }^{*} V_{12}+V_{21}{ }^{*} V_{22}\right) V_{22}{ }^{-1} \\
& =0
\end{aligned}
$$

by (37) and (21). Hence $\Lambda(V)_{12}$ has all the desired properties, and one readily checks (using $P_{\text {ker }} V_{22} *=$ $P_{2}-V_{22} V_{22}{ }^{-1}$ ) that the corresponding solution of (32) and (34)-(36) is given by (38). 
To find the general form of $H_{12}$, we make the ansatz $H_{12}=\Lambda(V)_{12}+h_{12}$. Then $H_{12}$ is an antisymmetric Hilbert-Schmidt operator if and only if $h_{12}$ is. It fulfills (41) if and only if $h_{12} V_{22}=0$, and we have $V_{21}\left(\operatorname{ker} V_{11}\right) \subset \operatorname{ker} H_{12}$ if and only if $V_{21}\left(\operatorname{ker} V_{11}\right) \subset \operatorname{ker} h_{12}$. The last two conditions are equivalent to $\left(\operatorname{ker} h_{12}\right)^{\perp} \subset\left(\operatorname{ran} V_{22} \oplus V_{21}\left(\operatorname{ker} V_{11}\right)\right)^{\perp}=\operatorname{ker} V_{22}{ }^{*} \ominus V_{21}\left(\operatorname{ker} V_{11}\right)$, and we then have by antisymmetry $\operatorname{ran} h_{12}=\Gamma\left(\operatorname{ran} h_{12}{ }^{*}\right) \subset \Gamma\left(\operatorname{ker} h_{12}\right)^{\perp} \subset \operatorname{ker} V_{11}{ }^{*} \ominus V_{12}\left(\operatorname{ker} V_{22}\right)$. As a result, the admissible components $H_{12}$ (as well as the remaining components (44)) have the form stated in the lemma. By (25), we may regard the $h_{12}$ as antisymmetric operators from one $m$-dimensional space to another, thus there are $\left(m^{2}-m\right) / 2$ linearly independent ones.

For $H_{12}=\Lambda(V)_{12}+h_{12}$ as above, we have $\left\|H_{12}\right\|_{2}^{2}=\left\|\Lambda(V)_{12}\right\|_{2}^{2}+\left\|h_{12}\right\|_{2}^{2}$. Hence the associate $\Lambda(V)$ is the antisymmetric solution of (34)-(36) with minimal Hilbert-Schmidt norm $\left\|\Lambda(V)_{12}\right\|_{2}$. In the rest of Section 1 , we shall work exclusively with $\Lambda(V)$. But we emphasize that all the results in Sections 4.7 and 4.5 hold as well if $\Lambda(V)$ is replaced everywhere by one of the operators $H$ described in Lemma 4.9. The only point where the choice of the associate (of $W$ below, not of $V$ ) is distinguished is in Section $4.3\left(\Lambda(W)_{12}=\right.$ 0 , see Lemma 4.8). We remark further that (38) reduces to the definition given by Ruijsenaars [8] (i.e. $\Lambda(V)_{12}=V_{12} V_{22}{ }^{-1}$ ) whenever $\omega_{P_{1}} \circ \varrho_{V}$ is pure (cf. Lemma 3.6 a)).

\subsection{Normal Form of Implementers}

As we have seen in Section 4.1, $: \exp (b(\Lambda(V)) / 2)$ : is (the quadratic form of) a densely defined operator with intertwining properties (34)-(36). To construct an isometric implementer for $\varrho_{V}$, let

$$
L_{V}:=\operatorname{dim} \operatorname{ker} V_{11}<\infty
$$

(see (23)) and choose an orthonormal basis $\left\{e_{1}, \ldots, e_{L_{V}}\right\}$ for ker $V_{11}$. For $r=1, \ldots, L_{V}$, set 9

$$
\begin{aligned}
& A_{r}:=a\left(e_{r}\right) \Psi(-\mathbf{1}), \\
& A_{V, r}:=a_{V}\left(e_{r}\right) \Psi(-\mathbf{1})=a\left(V_{12} \Gamma e_{r}\right)^{*} \Psi(-\mathbf{1})
\end{aligned}
$$

where $\Psi(-\mathbf{1})$ is the self-adjoint unitary implementer for $\alpha_{-1}$ with $\Psi(-\mathbf{1}) \Omega_{P_{1}}=\Omega_{P_{1}}$ (cf. the proof of Lemma 2.3). Then the $A_{r}{ }^{(*)}, A_{V, r}{ }^{*}$ respectively fulfill the CAR. Let $\mathcal{P}_{L_{V}}$ denote the index set consisting of pairs $(\sigma, s)$ with $s \in\left\{0, \ldots, L_{V}\right\}$ and $\sigma$ a permutation of order $L_{V}$ satisfying $\sigma(1)<\ldots<\sigma(s)$ and $\sigma(s+1)<\ldots<\sigma\left(L_{V}\right)$. $\mathcal{P}_{L_{V}}$ is canonically isomorphic to the power set $\mathfrak{P}_{L_{V}}$ of $\left\{1, \ldots, L_{V}\right\}$ through identification of $(\sigma, s)$ with $\{\sigma(1), \ldots, \sigma(s)\}$, hence its cardinality is $2^{L_{V}}$. We now define the following operator on $\mathcal{D}$

$$
\begin{aligned}
\Psi_{0}(V):= & \left(\operatorname{det}\left(P_{1}+\Lambda(V)_{12} \Lambda(V)_{12}{ }^{*}\right)\right)^{-1 / 4} . \\
& \cdot \sum_{(\sigma, s) \in \mathcal{P}_{L_{V}}}(-1)^{s} \operatorname{sign} \sigma A_{V, \sigma(1)} \cdots A_{V, \sigma(s)}: \exp (b(\Lambda(V)) / 2): A_{\sigma(s+1)} \cdots A_{\sigma\left(L_{V}\right)}
\end{aligned}
$$

with range contained in the space of $C^{\infty}$-vectors for the number operator.

Lemma 4.3 $\Psi_{0}(V)$ has a continuous extension to an isometry (denoted by the same symbol) on $\mathcal{F}_{a}\left(\mathcal{K}_{1}\right)$ with

$$
\Psi_{0}(V) \pi_{P_{1}}(A)=\pi_{P_{1}}\left(\varrho_{V}(A)\right) \Psi_{0}(V), \quad A \in \mathcal{C}(\mathcal{K}, \Gamma) .
$$

\footnotetext{
${ }^{g}$ It is also possible to incorporate the factors $\Psi(-\mathbf{1})$ into $: \exp (b(\Lambda(V)) / 2)$ : as is done in [8, 16], but the choice (45) simplifies the combinatorics.
} 
Proof. We first show

$$
\Psi_{0}(V) a(f)^{(*)}=a_{V}(f)^{(*)} \Psi_{0}(V), \quad f \in \mathcal{K}_{1}
$$

on $\mathcal{D}$. To this end, let us introduce the analog of Ruijsenaars' operator $\hat{\Gamma}(V)$ ]

$$
\hat{\Psi}(V):=\left(\operatorname{det}\left(P_{1}+\Lambda(V)_{12} \Lambda(V)_{12}{ }^{*}\right)\right)^{-1 / 4}: \exp (b(\Lambda(V)) / 2): .
$$

For $f \in\left(\operatorname{ker} V_{11}\right)^{\perp}$, (48) follows from (34), (35) together with $\left[a(f)^{(*)}, A_{r}\right]=\left[a_{V}(f)^{(*)}, A_{V, r}\right]=0$. To prove (48) for $f=e_{r}, r=1, \ldots, L_{V}$, we make use of

$$
\begin{aligned}
& {\left[a\left(e_{r}\right), A_{s}\right]=\left[a_{V}\left(e_{r}\right), A_{V, s}\right]=0,} \\
& {\left[a\left(e_{r}\right)^{*}, A_{s}\right]=\left[a_{V}\left(e_{r}\right)^{*}, A_{V, s}\right]=\delta_{r s} \Psi(-\mathbf{1}),} \\
& \left\{\Psi(-\mathbf{1}), A_{s}\right\}=\left\{\Psi(-\mathbf{1}), A_{V, s}\right\}=[\Psi(-\mathbf{1}), \hat{\Psi}(V)]=0 .
\end{aligned}
$$

Note further that for fixed $r$, the bijection $\left\{\mathcal{M} \in \mathfrak{P}_{L_{V}} \mid r \in \mathcal{M}\right\} \rightarrow\left\{\mathcal{M}^{\prime} \in \mathfrak{P}_{L_{V}} \mid r \notin \mathcal{M}^{\prime}\right\}, \mathcal{M} \mapsto \mathcal{M} \backslash\{r\}$ induces a bijection $(\sigma, s) \mapsto\left(\sigma^{\prime}, s^{\prime}\right)$ from $\left\{(\sigma, s) \in \mathcal{P}_{L_{V}} \mid r \in\{\sigma(1), \ldots, \sigma(s)\}\right\}$ onto $\left\{\left(\sigma^{\prime}, s^{\prime}\right) \in \mathcal{P}_{L_{V}} \mid r \notin\right.$ $\left.\left\{\sigma^{\prime}(1), \ldots, \sigma^{\prime}\left(s^{\prime}\right)\right\}\right\}$ with 16

$$
s=s^{\prime}+1, \quad(-1)^{s} \operatorname{sign} \sigma=(-1)^{r} \operatorname{sign} \sigma^{\prime}, \quad \sigma^{-1}(r)+\sigma^{\prime-1}(r)=r+s .
$$

We now have by virtue of the CAR, (50), (52) and (53) on $\mathcal{D}$

$$
\begin{aligned}
\Psi_{0}(V) a\left(e_{r}\right)= & A_{V, r} \sum_{\substack{(\sigma, s) \in \mathcal{P}_{L_{V}} \\
r \in\{\sigma(1), \ldots, \sigma(s)\}}}(-1)^{s+\sigma^{-1}(r)-1} \operatorname{sign} \sigma A_{V, \sigma(1)} \cdots \widetilde{A_{V, r}} \cdots A_{V, \sigma(s)} \cdot \\
= & a_{V}\left(e_{r}\right) \Psi(-\mathbf{1})^{2} \sum_{\substack{\left(\sigma^{\prime}, s^{\prime}\right) \in \mathcal{P}_{L_{V}} \\
r \notin\left\{\sigma^{\prime}(1), \ldots, \sigma^{\prime}\left(s^{\prime}\right)\right\}}}(-1)^{s^{\prime}} \operatorname{sign} \sigma^{\prime} A_{V, \sigma^{\prime}(1)} \cdots A_{V, \sigma^{\prime}\left(s^{\prime}\right)} \hat{\Psi}(V) A_{\sigma^{\prime}\left(s^{\prime}+1\right)} \cdots A_{\sigma^{\prime}\left(L_{V}\right)} \\
= & a_{V}\left(e_{r}\right) \Psi_{0}(V) .
\end{aligned}
$$

As a consequence of (34) and (36), we have $\hat{\Psi}(V) a\left(e_{r}\right)^{*}=a_{V}\left(e_{r}\right)^{*} \hat{\Psi}(V)=0$. This yields in connection with (51), 52) and (53)

$$
\begin{aligned}
& a_{V}\left(e_{r}\right)^{*} \Psi_{0}(V)= \\
& =\Psi(-\mathbf{1}) \sum_{\substack{(\sigma, s) \in \mathcal{P}_{L_{V}} \\
r \in\{\sigma(1), \ldots, \sigma(s)\}}}(-1)^{s+\sigma^{-1}(r)-1} \operatorname{sign} \sigma A_{V, \sigma(1)} \cdots \widetilde{A_{V, r}} \cdots A_{V, \sigma(s)} \hat{\Psi}(V) A_{\sigma(s+1)} \cdots A_{\sigma\left(L_{V}\right)} \\
& =\Psi(-\mathbf{1}) \sum_{\substack{\left(\sigma^{\prime}, s^{\prime}\right) \in \mathcal{P}_{L_{V}} \\
r \notin\left\{\sigma^{\prime}(1), \ldots, \sigma^{\prime}\left(s^{\prime}\right)\right\}}}(-1)^{s^{\prime}+\sigma^{\prime-1}(r)} \operatorname{sign} \sigma^{\prime} A_{V, \sigma^{\prime}(1)} \cdots A_{V, \sigma^{\prime}\left(s^{\prime}\right)} \hat{\Psi}(V) A_{\sigma^{\prime}\left(s^{\prime}+1\right)} \cdots \widetilde{A_{r}} \cdots A_{\sigma^{\prime}\left(L_{V}\right)} \\
& =\Psi_{0}(V) a\left(e_{r}\right)^{*},
\end{aligned}
$$

so (48) holds.

Since the $A_{V, r}{ }^{*}$ fulfill the CAR and $A_{V, r}{ }^{*} \hat{\Psi}(V)=0$, Ruijsenaars' result (33) implies

$$
\begin{aligned}
\left\|\Psi_{0}(V) \Omega_{P_{1}}\right\|^{2} & =\left\|A_{V, 1} \cdots A_{V, L_{V}} \hat{\Psi}(V) \Omega_{P_{1}}\right\|^{2} \\
& =\left\langle\hat{\Psi}(V) \Omega_{P_{1}}, A_{V, L_{V}}{ }^{*} A_{V, L_{V}} \cdots A_{V, 1}{ }^{*} A_{V, 1} \hat{\Psi}(V) \Omega_{P_{1}}\right\rangle \\
& =\left\|\hat{\Psi}(V) \Omega_{P_{1}}\right\|^{2} \\
& =1 .
\end{aligned}
$$


Since the $a_{V}(f)^{(*)}$ also fulfill the CAR and since $a_{V}(f) \Psi_{0}(V) \Omega_{P_{1}}=0$ by (48), we obtain for $g_{1}, \ldots, g_{m}, h_{1}, \ldots, h_{n} \in \mathcal{K}_{1}$

$$
\begin{aligned}
& \left\langle\Psi_{0}(V) a\left(g_{1}\right)^{*} \cdots a\left(g_{m}\right)^{*} \Omega_{P_{1}}, \Psi_{0}(V) a\left(h_{1}\right)^{*} \cdots a\left(h_{n}\right)^{*} \Omega_{P_{1}}\right\rangle= \\
& \quad=\left\langle\Psi_{0}(V) \Omega_{P_{1}}, a_{V}\left(g_{m}\right) \cdots a_{V}\left(g_{1}\right) a_{V}\left(h_{1}\right)^{*} \cdots a_{V}\left(h_{n}\right)^{*} \Psi_{0}(V) \Omega_{P_{1}}\right\rangle \\
& \quad=\left\langle a\left(g_{1}\right)^{*} \cdots a\left(g_{m}\right)^{*} \Omega_{P_{1}}, a\left(h_{1}\right)^{*} \cdots a\left(h_{n}\right)^{*} \Omega_{P_{1}}\right\rangle .
\end{aligned}
$$

Hence $\Psi_{0}(V)$ is isometric on $\mathcal{D}$ and has a continuous extension to an isometry which satisfies (48) on $\mathcal{F}_{a}\left(\mathcal{K}_{1}\right)$. But this implies $\Psi_{0}(V) \pi_{P_{1}}(A)=\pi_{P_{1}}\left(\varrho_{V}(A)\right) \Psi_{0}(V)$ for $A \in \mathcal{C}(\mathcal{K}, \Gamma)$.

We proceed to construct a complete set of implementers with the help of $\Psi_{0}(V)$. In view of the remark above Lemma 3.2, we have to look for partial isometries in $\pi_{P_{1}}\left(\varrho_{V}(\mathcal{C}(\mathcal{K}, \Gamma))\right)^{\prime}$ which contain $\operatorname{ran} \Psi_{0}(V)$ in their initial spaces. Since $\mathcal{K}$ is infinite dimensional, we have [12] $\pi_{P_{1}}\left(\varrho_{V}(\mathcal{C}(\mathcal{K}, \Gamma))\right)^{\prime}=\psi\left(\operatorname{ker} V^{*}\right)^{\prime \prime}$ with

$$
\psi(k):=\pi_{P_{1}}(B(k)) \Psi(-\mathbf{1}) .
$$

Lemma 4.4 Let $k \in \operatorname{ker} V^{*}$. Then $\psi(k)$ is a partial isometry with $\operatorname{ran} \Psi_{0}(V) \subset(\operatorname{ker} \psi(k))^{\perp}$ if and only if a) or b) below holds.

a) $\|k\|=\sqrt{2},|\langle k, \Gamma k\rangle|=2$. In this case, $\psi(k)$ is unitary.

b) $\|k\|=1, k \in \operatorname{ran}\left(P_{1}-\Lambda(V)_{12}{ }^{*}\right)$. In this case, $\mathcal{F}_{a}\left(\mathcal{K}_{1}\right)=(\operatorname{ker} \psi(k))^{\perp} \oplus \operatorname{ran} \psi(k)$.

Proof. Let $k \in \mathcal{K} . \psi(k)^{*} \psi(k)$ and $\psi(k) \psi(k)^{*}$ are projections if and only if one of the following holds:
1) $k=0$,
2) $\|k\|^{2}=|\langle k, \Gamma k\rangle|=2$,
3) $\|k\|=1,\langle k, \Gamma k\rangle=0$.

In the second case we have $|\langle k, \Gamma k\rangle|=\|k\| \cdot\|\Gamma k\|$, hence there exists $z \in U(1)$ with $\Gamma k=z k$. This implies $B(k)^{*} B(k)=B(\Gamma k) B(k)=\frac{z}{2}\{B(k), B(k)\}=\frac{z}{2}\langle\Gamma k, k\rangle \mathbf{1}=\mathbf{1}$ and $B(k) B(k)^{*}=\|k\|^{2} \mathbf{1}-B(k)^{*} B(k)=\mathbf{1}$, thus $\psi(k)$ is unitary.

In the third case we have $\psi(k)^{*} \psi(k)=\mathbf{1}-\psi(k) \psi(k)^{*}$, hence initial and final space of $\psi(k)$ are orthogonal to each other and sum up to $\mathcal{F}_{a}\left(\mathcal{K}_{1}\right)$. The requirement $\operatorname{ran} \Psi_{0}(V) \subset(\operatorname{ker} \psi(k))^{\perp}=\operatorname{ker} \psi(k)^{*}$ holds for $k \in \operatorname{ker} V^{*}$ if and only if $\psi(k)^{*} \Psi_{0}(V) \Omega_{P_{1}}=0$. This follows from $\psi(k)^{*} \in \pi_{P_{1}}\left(\varrho_{V}(\mathcal{C}(\mathcal{K}, \Gamma))\right)^{\prime}$ and the fact that vectors of the form $\Psi_{0}(V) \pi_{P_{1}}(A) \Omega_{P_{1}}=\pi_{P_{1}}\left(\varrho_{V}(A)\right) \Psi_{0}(V) \Omega_{P_{1}}, A \in \mathcal{C}(\mathcal{K}, \Gamma)$, are dense in $\operatorname{ran} \Psi_{0}(V)$. By $\left\{\psi(k)^{*}, A_{V, r}\right\}=0$, we further have (cf. the proof of Lemma 4.3) $\left\|\psi(k)^{*} \Psi_{0}(V) \Omega_{P_{1}}\right\|=$ $\left\|\psi(k)^{*} \hat{\Psi}(V) \Omega_{P_{1}}\right\|$. By Lemma 4.1 and (52),

$$
\psi(k)^{*} \hat{\Psi}(V) \Omega_{P_{1}}=-\left(a\left(P_{1} k\right)+a\left(P_{1} \Gamma k\right)^{*}\right) \hat{\Psi}(V) \Omega_{P_{1}}=-a\left(\left(P_{1}-\Lambda(V)_{12}\right) \Gamma k\right)^{*} \hat{\Psi}(V) \Omega_{P_{1}}
$$

which vanishes if and only if $k \in \operatorname{ker}\left(P_{1}-\Lambda(V)_{12}\right) \Gamma=\operatorname{ran}\left(P_{1}-\Lambda(V)_{12}{ }^{*}\right)$ (cf. (59) below). But for such $k$, $\langle k, \Gamma k\rangle=0$ automatically holds (see Section 4.3 ), so we conclude that partial isometries $\psi(k)$ of type 3) with $k \in \operatorname{ker} V^{*}$ and $\operatorname{ran} \Psi_{0}(V) \subset(\operatorname{ker} \psi(k))^{\perp}$ are completely characterized by condition b).

For our purposes, the partial isometries described in part b) of the lemma are the important ones. Let $\left\{k_{1}, \ldots, k_{m}\right\}$ be an orthonormal basis for $\operatorname{ker} V^{*} \cap \operatorname{ran}\left(P_{1}-\Lambda(V)_{12}{ }^{*}\right)$. For $\beta=\left(\beta_{1}, \ldots, \beta_{r}\right) \in I_{m}$ (cf. (5) and (17)), set

$$
\begin{aligned}
& \psi_{\beta}:=\psi\left(k_{\beta_{1}}\right) \cdots \psi\left(k_{\beta_{r}}\right), \\
& \Psi_{\beta}(V):=\psi_{\beta} \Psi_{0}(V) .
\end{aligned}
$$

Since the $\left(\psi_{j}^{(*)}\right)_{j=1, \ldots, m}$ fulfill the CAR

$$
\left\{\psi_{j}, \psi_{l}\right\}=\left\{\psi_{j}^{*}, \psi_{l}^{*}\right\}=0, \quad\left\{\psi_{j}, \psi_{l}^{*}\right\}=\delta_{j l} \mathbf{1},
$$

the $\psi_{\beta}$ are partial isometries in $\pi_{P_{1}}\left(\varrho_{V}(\mathcal{C}(\mathcal{K}, \Gamma))\right)^{\prime}$ with $\operatorname{ran} \Psi_{0}(V) \subset\left(\operatorname{ker} \psi_{\beta}\right)^{\perp}$. 
Theorem $4.5 \operatorname{ker} V^{*} \cap \operatorname{ran}\left(P_{1}-\Lambda(V)_{12}{ }^{*}\right)$ has dimension $m=\frac{1}{2}$ ind $V^{*}$, and the $d_{V}=2^{m}$ isometries $\left(\Psi_{\beta}(V)\right)_{\beta \in I_{m}}$ implement $\varrho_{V}$ in $\pi_{P_{1}}$ in the sense of Definition 3.1 .

Proof. As a consequence of (55) and $\psi_{j}^{*} \Psi_{0}(V)=\Psi_{0}(V)^{*} \psi_{j}=0$, the first equation in (11) holds:

$$
\Psi_{\beta}(V)^{*} \Psi_{\gamma}(V)=\Psi_{0}(V)^{*} \psi_{\beta_{r}}^{*} \cdots \psi_{\beta_{1}}^{*} \psi_{\gamma_{1}} \cdots \psi_{\gamma_{s}} \Psi_{0}(V)=\delta_{\beta \gamma} \mathbf{1}
$$

for $\beta=\left(\beta_{1}, \ldots, \beta_{r}\right), \gamma=\left(\gamma_{1}, \ldots, \gamma_{s}\right) \in I_{m}$. Clearly, the $\Psi_{\beta}(V)$ have the intertwining property (14)

$$
\Psi_{\beta}(V) \pi_{P_{1}}(A)=\pi_{P_{1}}\left(\varrho_{V}(A)\right) \Psi_{\beta}(V), \quad A \in \mathcal{C}(\mathcal{K}, \Gamma)
$$

since $\psi_{\beta} \in \pi_{P_{1}}\left(\varrho_{V}(\mathcal{C}(\mathcal{K}, \Gamma))\right)^{\prime}$. We postpone the proofs of the completeness relation

$$
\sum_{\beta \in I_{m}} \Psi_{\beta}(V) \Psi_{\beta}(V)^{*}=\mathbf{1}
$$

and of $m=\frac{1}{2}$ ind $V^{*}$ to Section 4.3. Since (57) and (58) imply (12), the theorem will then be proven.

By (55) and by $\psi_{j}^{*} \Psi_{0}(V)=0, j=1, \ldots, m$, the $\psi_{j}$ may be regarded as creation operators relative to the vacuum $\Psi_{0}(V)$. The Hilbert space spanned by the $\Psi_{\beta}(V)$ is therefore canonically isomorphic to the antisymmetric Fock space over $\operatorname{ker} V^{*} \cap \operatorname{ran}\left(P_{1}-\Lambda(V)_{12}{ }^{*}\right)$.

\subsection{Decomposition of Bogoliubov Operators and Proof of Completeness}

Let us first remark that $m:=\operatorname{dim}\left(\operatorname{ker} V^{*} \cap \operatorname{ran}\left(P_{1}-\Lambda(V)_{12}{ }^{*}\right)\right)=\frac{1}{2}$ ind $V^{*}$ implies completeness (58) in the case of finite index since the representation $\pi_{P_{1}} \circ \varrho_{V}$ splits into $d_{V}$ irreducibles by Theorem 3.7 and since the ranges of the isometries $\Psi_{\beta}(V)$ are mutually orthogonal, irreducible subspaces for $\pi_{P_{1}} \circ \varrho_{V}$. However, we shall give a different proof of completeness which also works in the case of infinite index. The goal is a (to a certain extent canonical) product decomposition $V=U W$ where $U \in \mathcal{I}_{P_{1}}^{0}(\mathcal{K}, \Gamma)$ is unitary and $W \in \mathcal{I}_{P_{1}}^{\text {ind } V^{*}}(\mathcal{K}, \Gamma)$ induces a pure and gauge invariant state $\omega_{P_{1}} \varrho_{W} . U$ and $W$ will be chosen such that $\Lambda(U)_{12}=\Lambda(V)_{12}$ and $\Lambda(W)_{12}=0$, and (58) will follow from completeness of implementers for $\varrho_{W}$ which in turn is a consequence of Lemma 3.2 .

We start with the proof of $m=\frac{1}{2}$ ind $V^{*}$.

Lemma 4.6 Let $T$ be an antisymmetric Hilbert-Schmidt operator from $\mathcal{K}_{1}$ to $\mathcal{K}_{2} . \quad$ Then $\left(\begin{array}{l}P_{1} \bar{T} \\ T P_{2}\end{array}\right)$ is a bijection on $\mathcal{K}$, and $\mathcal{K}=\operatorname{ran}\left(P_{1}+T\right) \oplus \operatorname{ran}\left(P_{2}+\bar{T}\right)$. If we set

$$
\begin{aligned}
P & :=\left(P_{1}+T\right)\left(P_{1}+T^{*} T\right)^{-1}\left(P_{1}+T^{*}\right), \\
U_{T} & :=\left(P_{1}+T\right)\left(P_{1}+T^{*} T\right)^{-1 / 2}+\left(P_{2}+\bar{T}\right)\left(P_{2}+T T^{*}\right)^{-1 / 2},
\end{aligned}
$$

then $P$ is a basis projection with $\operatorname{ran} P=\operatorname{ran}\left(P_{1}+T\right)$ and $P_{2} P \in \mathfrak{J}_{2}(\mathcal{K})$, and $U_{T} \in \mathcal{I}_{P_{1}}^{0}(\mathcal{K}, \Gamma)$ is unitary with $U_{T}^{*} P U_{T}=P_{1}$.

Proof. Let $k \in \operatorname{ker}(\mathbf{1}+T+\bar{T})$. Then $P_{1} k=-\bar{T} P_{2} k$ and $P_{2} k=-T P_{1} k$, hence $\left(P_{1}+T^{*} T\right) P_{1} k=0$ by antisymmetry (32). But $P_{1}+T^{*} T$ is a bijection on $\mathcal{K}_{1}$, so $k=0$ and $\mathbf{1}+T+\bar{T}$ is injective. Since $\mathbf{1}+T+\bar{T}$ is Fredholm with vanishing index by compactness of $T$, it is also surjective.

Let $f_{j} \in \mathcal{K}_{j}, j=1,2$. Then $\left\langle\left(P_{1}+T\right) f_{1},\left(P_{2}+\bar{T}\right) f_{2}\right\rangle=\left\langle f_{1}, \bar{T} f_{2}\right\rangle+\left\langle T f_{1}, f_{2}\right\rangle=0$ by antisymmetry which proves $\mathcal{K}=\operatorname{ran}\left(P_{1}+T\right) \oplus \operatorname{ran}\left(P_{2}+\bar{T}\right)$. It is not hard to see that $P$ is the projection onto $\operatorname{ran}\left(P_{1}+T\right)$ and therefore a basis projection. The unitary $U_{T}$ results from polar decomposition of $\mathbf{1}+T+\bar{T}=U_{T}|\mathbf{1}+T+\bar{T}|$ (by the way, $U_{T}$ coincides with Araki's canonical choice of a Bogoliubov 
operator that transforms $P$ into $P_{1}$ [15]). $P_{2} P=T\left(P_{1}+T^{*} T\right)^{-1}\left(P_{1}+T^{*}\right)$ and $\left(U_{T}\right)_{21}=T\left(P_{1}+T^{*} T\right)^{-1 / 2}$ are Hilbert-Schmidt since $T$ is, and $P U_{T}=\left(P_{1}+T\right)\left(P_{1}+T^{*} T\right)^{-1 / 2}=U_{T} P_{1}$.

Application of Lemma 4.6 to $T=\overline{\Lambda(V)_{12}}=-\Lambda(V)_{12}{ }^{*}$ yields the basis projection $P_{V}$ with $\operatorname{ran} P_{V}=$ $\operatorname{ran}\left(P_{1}-\Lambda(V)_{12}{ }^{*}\right)$.

Lemma 4.7 $P_{V}$ commutes with $V V^{*}$. As a consequence, $\operatorname{ker} V^{*}=P_{V}\left(\operatorname{ker} V^{*}\right) \oplus \overline{P_{V}}\left(\operatorname{ker} V^{*}\right)$ and $m=$ $\operatorname{dim} P_{V}\left(\operatorname{ker} V^{*}\right)=\frac{1}{2}$ ind $V^{*}$.

Proof. $\left[P_{V}, V V^{*}\right]=0$ is equivalent to $\left[\overline{P_{V}}, V V^{*}\right]=0$ or to $V V^{*}\left(\operatorname{ran}\left(P_{2}+\Lambda(V)_{12}\right)\right) \subset \operatorname{ker}\left(P_{1}-\Lambda(V)_{12}\right)$ since

$$
\operatorname{ran} \overline{P_{V}}=\operatorname{ran}\left(P_{2}+\Lambda(V)_{12}\right)=\operatorname{ker} P_{V}=\operatorname{ker}\left(P_{1}-\Lambda(V)_{12}\right) .
$$

By definition, $\Lambda(V)_{12}$ fulfills $\Lambda(V)_{12} V_{22}=V_{12} P_{\operatorname{ran} V_{22}}{ }^{*}$ and $\Lambda(V)_{12} V_{21} P_{\text {ker } V_{11}}=0$. Antisymmetry of

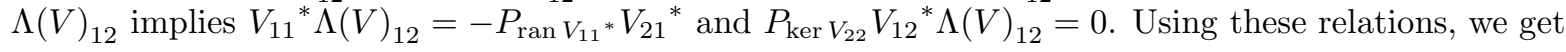

$$
\begin{aligned}
& \left(P_{1}-\Lambda(V)_{12}\right) V V^{*}\left(P_{2}+\Lambda(V)_{12}\right)=V_{11} V_{21}{ }^{*}+V_{12} V_{22}{ }^{*}+\left(V_{11} V_{11}{ }^{*}+V_{12} V_{12}{ }^{*}\right) \Lambda(V)_{12}-\Lambda(V)_{12} \text {. } \\
& \cdot\left(V_{21} V_{21}{ }^{*}+V_{22} V_{22}{ }^{*}\right)-\Lambda(V)_{12}\left(V_{22} V_{12}{ }^{*}+V_{21} V_{11}{ }^{*}\right) \Lambda(V)_{12} \\
& =V_{11} V_{21}{ }^{*}+V_{12} V_{22}{ }^{*}-V_{11} V_{21}{ }^{*}+V_{12} V_{12}{ }^{*} \Lambda(V)_{12}-\Lambda(V)_{12} V_{21} V_{21}{ }^{*} \\
& -V_{12} V_{22}{ }^{*}-V_{12} P_{\operatorname{ran} V_{22}} V_{12}{ }^{*} \Lambda(V)_{12}+\Lambda(V)_{12} V_{21} P_{\operatorname{ran} V_{11}} * V_{21}{ }^{*} \\
& =V_{12} P_{\text {ker } V_{22}} V_{12}{ }^{*} \Lambda(V)_{12}-\Lambda(V)_{12} V_{21} P_{\text {ker } V_{11}} V_{21}{ }^{*} \\
& =0 \text {. }
\end{aligned}
$$

Next we present a distinguished choice of $W$ for the product decomposition $V=U W$. Namely, let $W_{11}$ be the partial isometry with $\operatorname{ker} W_{11}{ }^{(*)}=\operatorname{ker} V_{11}{ }^{(*)}$ appearing in the polar decomposition of $V_{11}$ :

$$
V_{11}=W_{11}\left|V_{11}\right|
$$

(the idea of using polar decomposition of $V_{11}$ stems from [23]). Set

$$
W_{21}:=V_{21} P_{\text {ker } V_{11}}
$$

then $W_{21}$ is a partial isometry with initial space $\operatorname{ker} V_{11}$ and final space $V_{21}\left(\operatorname{ker} V_{11}\right) \subset \operatorname{ker} V_{22}{ }^{*}(\mathrm{cf}$. (24)), and set $W_{12}:=\overline{W_{21}}, W_{22}:=\overline{W_{11}}$.

Lemma 4.8 $W$ defined above has the following properties:

a) $W \in \mathcal{I}_{P_{1}}(\mathcal{K}, \Gamma)$, $\operatorname{ker} W^{*}=\left(\operatorname{ker} V_{11}{ }^{*} \ominus V_{12}\left(\operatorname{ker} V_{22}\right)\right) \oplus\left(\operatorname{ker} V_{22}{ }^{*} \ominus V_{21}\left(\operatorname{ker} V_{11}\right)\right)$ and ind $W=\operatorname{ind} V$;

b) $S_{W}:=W^{*} P_{1} W$ is the projection onto $\left(\operatorname{ker} V_{11}\right)^{\perp} \oplus \operatorname{ker} V_{22}$, and $\omega_{S_{W}}$ is pure and gauge invariant;

c) $\Lambda(W)_{12}=0$.

Proof. a) $W$ is clearly a Bogoliubov operator with $\operatorname{ker} W^{*}=\left(\operatorname{ker} V_{11}{ }^{*} \ominus V_{12}\left(\operatorname{ker} V_{22}\right)\right) \oplus\left(\operatorname{ker} V_{22}{ }^{*} \ominus\right.$ $V_{21}\left(\operatorname{ker} V_{11}\right)$ ). Therefore ind $W^{*}=\operatorname{dim} \operatorname{ker} W^{*}=2$ ind $V_{11}{ }^{*}=\operatorname{ind} V^{*}\left(\operatorname{cf}\right.$. (25)), and $W \in \mathcal{I}_{P_{1}}(\mathcal{K}, \Gamma)$ since $W_{12}$ has finite rank.

b) By a straightforward computation, $S_{W}$ is the projection onto $\left(\operatorname{ker} V_{11}\right)^{\perp} \oplus \operatorname{ker} V_{22} \cdot \omega_{S_{W}}=\omega_{P_{1}} \circ \varrho_{W}$ is pure by Lemma 3.6 a) and gauge invariant by $\left[P_{1}, S_{W}\right]=0$ (cf. Section [2).

c) $\Lambda(W)_{12}=0$ follows from $W_{11}{ }^{-1}=W_{11}{ }^{*}$ and $W_{12} W_{22}{ }^{*}=W_{11} W_{21}{ }^{*}=0$.

It remains to specify the factor $U$ in $V=U W$. $U$ has necessarily the form $U=V W^{*}+u$ where $u=\bar{u}$ is a partial isometry with initial space $\operatorname{ker} W^{*}=P_{1}\left(\operatorname{ker} W^{*}\right) \oplus P_{2}\left(\operatorname{ker} W^{*}\right)$ and final space $\operatorname{ker} V^{*}=$ 
$P_{V}\left(\operatorname{ker} V^{*}\right) \oplus \overline{P_{V}}\left(\operatorname{ker} V^{*}\right)$. We may choose $u$ such that $u P_{1}=P_{V} u$ (for example, suitable $u P_{1}$ is obtained by polar decomposition of $R_{V}$ below). Then $P_{2} P_{V} \in \mathfrak{J}_{2}(\mathcal{K})$ implies that $u_{21}=P_{2} P_{V} u$ and $U_{21}=$ $V_{21} W_{11}{ }^{*}+u_{21}$ are Hilbert-Schmidt. We need the following lemma to exhibit further properties of $U$. Remember that $Q_{W}=\mathbf{1}-W W^{*}$ denotes the projection onto ker $W^{*}$.

Lemma 4.9 $R_{V}:=\left(P_{1}-\Lambda(V)_{12}{ }^{*}\right)\left(P_{1}+V_{11} V_{21}{ }^{*} \Lambda(V)_{12}{ }^{*}\right) P_{1} Q_{W}$ maps $P_{1}\left(\operatorname{ker} W^{*}\right)$ bijectively onto $P_{V}\left(\operatorname{ker} V^{*}\right)$.

Proof. By Lemma 4.7, $P_{V}\left(\operatorname{ker} V^{*}\right)=\operatorname{ker}\left(P_{2}+\Lambda(V)_{12}{ }^{*}\right) \cap \operatorname{ker} V^{*}$. Hence $k \in P_{V}\left(\operatorname{ker} V^{*}\right)$ if and only if $P_{2} k=-\Lambda(V){ }_{12}{ }^{*} P_{1} k, V_{11}{ }^{*} P_{1} k+V_{21}{ }^{*} P_{2} k=0$ and $V_{22}{ }^{*} P_{2} k+V_{12}{ }^{*} P_{1} k=0$. Thus $P_{2} k$ is determined by $P_{1} k$, and $P_{1} k$ has to satisfy

$$
P_{1} k \in \operatorname{ker}\left(V_{11}{ }^{*}-V_{21}{ }^{*} \Lambda(V)_{12}{ }^{*}\right) \cap \operatorname{ker}\left(V_{12}{ }^{*}-V_{22}{ }^{*} \Lambda(V)_{12}{ }^{*}\right) .
$$

$\Lambda(V)_{12} V_{22}=V_{12} P_{\operatorname{ran} V_{22}}$ implies $\operatorname{ker}\left(V_{12}{ }^{*}-V_{22}{ }^{*} \Lambda(V)_{12}{ }^{*}\right)=\operatorname{ran}\left(V_{12}-V_{12} P_{\operatorname{ran} V_{22}{ }^{*}}\right)^{\perp}=\left(V_{12}\left(\operatorname{ker} V_{22}\right)\right)^{\perp}$. Since

$$
\mathcal{K}_{1}=P_{1}\left(\operatorname{ker} W^{*}\right) \oplus \operatorname{ran} V_{11} \oplus V_{12}\left(\operatorname{ker} V_{22}\right)
$$

(cf. Lemma 4.8), we may write $P_{1} k=f+g, f \in P_{1}\left(\operatorname{ker} W^{*}\right), g \in \operatorname{ran} V_{11}$. We then have $V_{21}{ }^{*} \Lambda(V)_{12}{ }^{*} g=$ $V_{21}{ }^{*} \Lambda(V)_{12}{ }^{*} V_{11} V_{11}{ }^{-1} g=-V_{21}{ }^{*} \Gamma \Lambda(V)_{12} V_{22} \Gamma V_{11}{ }^{-1} g=-V_{21}{ }^{*} V_{21} P_{\operatorname{ran}} V_{11}{ }^{*} V_{11}{ }^{-1} g=\left(V_{11}{ }^{*}-V_{11}{ }^{-1}\right) g$ by (37) and (19). Hence the condition $P_{1} k \in \operatorname{ker}\left(V_{11}{ }^{*}-V_{21}{ }^{*} \Lambda(V)_{12}{ }^{*}\right)$ is equivalent to $V_{11}{ }^{-1} g=V_{21}{ }^{*} \Lambda(V)_{12}{ }^{*} f$ or to $g=V_{11} V_{21}{ }^{*} \Lambda(V)_{12}{ }^{*} f$ (since $\left.P_{\text {ker } V_{11}} V_{21}{ }^{*} \Lambda(V)_{12}{ }^{*}=0\right)$ ). As a result, $k \in P_{V}\left(\operatorname{ker} V^{*}\right)$ if and only if there exists $f \in P_{1}\left(\operatorname{ker} W^{*}\right)$ such that $P_{1} k=\left(P_{1}+V_{11} V_{21}{ }^{*} \Lambda(V)_{12}{ }^{*}\right) f$ and $P_{2} k=-\Lambda(V)_{12}{ }^{*} P_{1} k$, i.e. $k=R_{V} f$. Hence $\operatorname{ran} R_{V}=P_{V}\left(\operatorname{ker} V^{*}\right)$.

To show that $R_{V}$ is one-to-one, assume that $f \in P_{1}\left(\operatorname{ker} W^{*}\right)$ and $R_{V} f=0$. Then $0=P_{1} Q_{W} R_{V} f=f$ since $P_{1} Q_{W} R_{V}=P_{1} Q_{W}$.

Proposition 4.10 Let $V \in \mathcal{I}_{P_{1}}(\mathcal{K}, \Gamma)$, and let $W \in \mathcal{I}_{P_{1}}(\mathcal{K}, \Gamma)$ be defined by (60) and (61) (with properties listed in Lemma 4.8). Then there exists $U \in \mathcal{I}_{P_{1}}^{0}(\mathcal{K}, \Gamma)$ with $U\left(P_{1}\left(\operatorname{ker} W^{*}\right)\right)=P_{V}\left(\operatorname{ker} V^{*}\right)$ and $V=U W$. Such $U$ fulfills $\operatorname{ker} U_{11}=\{0\}$ and $\Lambda(U)_{12}=\Lambda(V)_{12}$.

Proof. It remains to prove the last two statements. We have $U_{11}=P_{V_{12}\left(\operatorname{ker} V_{22}\right)}+\left|V_{11}{ }^{*}\right|+u_{11}$ by definition of $W$, and $\operatorname{ran} u_{11}=\operatorname{ran} P_{1} P_{V} u=\operatorname{ran} P_{1} R_{V} \subset P_{1}\left(\operatorname{ker} W^{*}\right) \oplus \operatorname{ran} V_{11}$ by $u P_{1}=P_{V} u$ and by Lemma 4.9 . This implies $\operatorname{ker} U_{11} \subset P_{1}\left(\operatorname{ker} W^{*}\right) \oplus \operatorname{ran} V_{11}$ (cf. (62)). Let $f \in P_{1}\left(\operatorname{ker} W^{*}\right), g \in \operatorname{ran} V_{11}$, and assume $0=U_{11}(f+g)=u_{11} f+\left|V_{11}{ }^{*}\right| g$. By Lemma 4.9, there exists $f^{\prime} \in P_{1}\left(\operatorname{ker} W^{*}\right)$ with $u P_{1} f=R_{V} f^{\prime}$. Then $0=P_{1} Q_{W} U_{11}(f+g)=P_{1} Q_{W} R_{V} f^{\prime}=f^{\prime}$, hence $f=0=g$. This proves ker $U_{11}=\{0\}$.

Let $f \in P_{1}\left(\operatorname{ker} W^{*}\right)$. Since there exists $f^{\prime} \in P_{1}\left(\operatorname{ker} W^{*}\right)$ with $u P_{1} f=R_{V} f^{\prime}$, we see that $u_{21} f=P_{2} R_{V} f^{\prime}=\overline{\Lambda(V)_{12}} P_{1} R_{V} f^{\prime}=\overline{\Lambda(V)_{12}} u_{11} f$ by definition of $R_{V}$. Hence $\Lambda(V)_{12} U_{22}=\left(V_{12} V_{22}{ }^{-1}-\right.$ $\left.V_{11}{ }^{-1 *} V_{21}{ }^{*} P_{\text {ker } V_{22}{ }^{*}}\right)\left(P_{V_{21}\left(\operatorname{ker} V_{11}\right)}+\left|V_{22}{ }^{*}\right|\right)+\Lambda(V)_{12} u_{22}=V_{12} V_{22}{ }^{-1}\left|V_{22}{ }^{*}\right|+u_{12}=U_{12}$ by (37) and $\left|V_{22}{ }^{*}\right|=V_{22} W_{22}{ }^{*}$. But this means $\Lambda(U)_{12}=\Lambda(V)_{12}$ (cf. the remark below Lemma 4.2).

The following result has already been obtained, in the case of finite index, in Lemma $3.4\left(S_{V}-S_{V^{\prime}}\right.$ is automatically Hilbert-Schmidt for $\left.V, V^{\prime} \in \mathcal{I}_{P_{1}}(\mathcal{K}, \Gamma)\right)$.

Corollary 4.11 The $\mathcal{I}_{P_{1}}^{0}(\mathcal{K}, \Gamma)$-orbits in $\mathcal{I}_{P_{1}}(\mathcal{K}, \Gamma)$ with respect to left multiplication are precisely the sets $\mathcal{I}_{P_{1}}^{2 m}(\mathcal{K}, \Gamma), m \in \mathbb{N} \cup\{\infty\}$.

Proof. Let $V, V^{\prime} \in \mathcal{I}_{P_{1}}^{2 m}(\mathcal{K}, \Gamma)$ be given, with decompositions $V=U W, V^{\prime}=U^{\prime} W^{\prime}$ as in Proposition 4.10 . Since $P_{1}$ leaves $\operatorname{ker} W^{\prime *}$ and ker $W^{*}$ invariant, we may choose a partial isometry $u^{\prime \prime}$ with initial space $\operatorname{ker} W^{\prime *}$ and final space $\operatorname{ker} W^{*}$ such that $\overline{u^{\prime \prime}}=u^{\prime \prime}$ and $\left[P_{1}, u^{\prime \prime}\right]=0$. Then $U^{\prime \prime}:=W W^{\prime *}+u^{\prime \prime} \in \mathcal{I}_{P_{1}}^{0}(\mathcal{K}, \Gamma)$ 
fulfills $U^{\prime \prime} W^{\prime}=W$. This implies $\left(U U^{\prime \prime} U^{\prime *}\right) V^{\prime}=V$, so $\mathcal{I}_{P_{1}}^{0}(\mathcal{K}, \Gamma)$ acts transitively on $\mathcal{I}_{P_{1}}^{2 m}(\mathcal{K}, \Gamma)$.

End of Proof of Theorem 4.5. Let $V=U W$ as in Proposition 4.10. We first apply the construction from Section 4.2 to $W$ and compute the values of implementers $\Psi_{\beta}(W)$ on $\Omega_{P}$. Since ker $W_{11}=\operatorname{ker} V_{11}$ and $\left.W_{12}\right|_{\operatorname{ker} V_{22}}=\left.V_{12}\right|_{\operatorname{ker} V_{22}}$, we may choose $A_{W, r}=A_{V, r}, r=1, \ldots, L_{V}\left(\right.$ cf. (45)). Then $\Psi_{0}(W) \Omega_{P_{1}}=$ $(-1)^{L_{V}} A_{V, 1} \cdots A_{V, L_{V}} \Omega_{P_{1}}$ by $\Lambda(W)_{12}=0$ (cf. (46)). According to Section 4.2, we have to choose an orthonormal basis $\left\{f_{1}, \ldots, f_{m}\right\}$ for $\operatorname{ker} W^{*} \cap \operatorname{ran}\left(P_{1}-\Lambda(W)_{12}{ }^{*}\right)=P_{1}\left(\operatorname{ker} W^{*}\right), m=\frac{1}{2}$ ind $W^{*}=\frac{1}{2}$ ind $V^{*}$, to obtain further implementers for $\varrho_{W}$. Since $U\left(P_{1}\left(\operatorname{ker} W^{*}\right)\right)=P_{V}\left(\operatorname{ker} V^{*}\right)=\operatorname{ker} V^{*} \cap \operatorname{ran}\left(P_{1}-\Lambda(V)_{12}{ }^{*}\right)$, we may choose the $f_{j}$ such that $U f_{j}=k_{j}, j=1, \ldots, m$. For a multi-index $\beta=\left(\beta_{1}, \ldots, \beta_{r}\right) \in I_{m}$, we have by definition (54)

$$
\Psi_{\beta}(W) \Omega_{P_{1}}=(-1)^{L_{V}} \psi\left(f_{\beta_{1}}\right) \cdots \psi\left(f_{\beta_{r}}\right) A_{V, 1} \cdots A_{V, L_{V}} \Omega_{P_{1}} .
$$

Let $A:=a_{W}\left(e_{L_{V}}\right)^{*} \cdots a_{W}\left(e_{1}\right)^{*} \in \pi_{P_{1}}\left(\varrho_{W}(\mathcal{C}(\mathcal{K}, \Gamma))\right)$ (cf. (45)). Remembering $\psi\left(f_{j}\right)=a\left(f_{j}\right)^{*} \Psi(-\mathbf{1}) \in$ $\pi_{P_{1}}\left(\varrho_{W}(\mathcal{C}(\mathcal{K}, \Gamma))\right)^{\prime}$ and neglecting signs, we get $A \Psi_{\beta}(W) \Omega_{P_{1}}= \pm a\left(f_{\beta_{1}}\right)^{*} \cdots a\left(f_{\beta_{r}}\right)^{*} \Omega_{P_{1}}= \pm \phi_{\beta}^{W}$ where $\phi_{\beta}^{W} \in \mathcal{F}_{\beta}^{W}$ is the cyclic vector defined in (17). Since $\omega_{P_{1}} \circ \varrho_{W}$ is pure, $\mathcal{F}_{\beta}^{W}$ is an irreducible subspace for $\pi_{P_{1}} \circ \varrho_{W}$. But by (56) and (57), $\operatorname{ran} \Psi_{\beta}(W)$ is also irreducible for $\pi_{P_{1}} \circ \varrho_{W}$. Since both spaces contain $\phi_{\beta}^{W}$, they must coincide. By Lemma 3.2, $\bigoplus_{\beta} \operatorname{ran} \Psi_{\beta}(W)=\mathcal{F}_{a}\left(\mathcal{K}_{1}\right)$, i.e. (58) holds for $W$.

Now let $\Psi_{0}(U)$ be the unitary implementer for $\alpha_{U}$ given by (46). Since $\varrho_{V}=\alpha_{U} \varrho_{W}$, the isometries $\left(\Psi_{0}(U) \Psi_{\beta}(W)\right)_{\beta \in I_{m}}$ implement $\varrho_{V}$ in $\pi_{P_{1}}$. We are going to show that actually

$$
\Psi_{0}(U) \Psi_{\beta}(W)=\Psi_{\beta}(V)
$$

holds under the above assumptions. Since each implementer is completely determined by its value on $\Omega_{P_{1}}$ (this follows from (57)), it suffices to prove (63) on $\Omega_{P_{1}}$. Note that $\left[\Psi_{0}(U), \Psi(-\mathbf{1})\right]=0$ since $\operatorname{ker} U_{11}=\{0\}$. Hence $\Psi_{0}(U) \psi\left(f_{j}\right)=\psi\left(k_{j}\right) \Psi_{0}(U)$ by $U f_{j}=k_{j}$, and $\left[\Psi_{0}(U), A_{V, r}\right]=0$ by $\left[\Psi_{0}(U), a_{V}\left(e_{r}\right)\right]=0$. Moreover, $\Lambda(U)_{12}=\Lambda(V)_{12}$ implies

$$
\Psi_{0}(U) \Omega_{P_{1}}=\hat{\Psi}(V) \Omega_{P_{1}}
$$

(see (49)), and we obtain

$$
\Psi_{0}(U) \Psi_{\beta}(W) \Omega_{P_{1}}=(-1)^{L_{V}} \psi\left(k_{\beta_{1}}\right) \cdots \psi\left(k_{\beta_{r}}\right) A_{V, 1} \cdots A_{V, L_{V}} \hat{\Psi}(V) \Omega_{P_{1}}=\Psi_{\beta}(V) \Omega_{P_{1}} .
$$

\section{Structure of the Semigroup of Implementable Endomor- phisms}

Let $P_{1}$ be a basis projection of $(\mathcal{K}, \Gamma)$ and $P_{2}:=\overline{P_{1}}$. It is easily seen that $\mathcal{I}_{P_{1}}(\mathcal{K}, \Gamma)$ is a topological semigroup relative to the metric (cf. [15])

$$
\delta_{P_{1}}\left(V, V^{\prime}\right):=\left\|V-V^{\prime}\right\|+\left\|V_{12}-V_{12}^{\prime}\right\|_{2} .
$$

The present section is devoted to the study of the connected components of $\mathcal{I}_{P_{1}}(\mathcal{K}, \Gamma)=\bigcup_{m} \mathcal{I}_{P_{1}}^{2 m}(\mathcal{K}, \Gamma)$. It is inspired by the work of Carey, Hurst and O'Brien [23].

Araki 15 has shown that the group $\mathcal{I}_{P_{1}}^{0}(\mathcal{K}, \Gamma) \subset \mathcal{I}_{P_{1}}(\mathcal{K}, \Gamma)$ consists of two connected components $\mathcal{I}_{P_{1}}^{0}(\mathcal{K}, \Gamma)^{ \pm}$. Namely,

$$
\chi(U):=(-1)^{\operatorname{dim} \operatorname{ker} U_{11}}
$$


defines a continuous character $\chi$ on $\mathcal{I}_{P_{1}}^{0}(\mathcal{K}, \Gamma)$, and $\left.\chi\right|_{\mathcal{I}_{P_{1}}^{0}(\mathcal{K}, \Gamma)^{ \pm}}= \pm 1$. However, we shall see that $\mathcal{I}_{P_{1}}^{2 m}(\mathcal{K}, \Gamma)$ is connected if $m \neq 0$ and that $\chi: V \mapsto(-1)^{\text {dim } \operatorname{ker} V_{11}}$ remains neither multiplicative nor continuous when extended to the whole semigroup $\mathcal{I}_{P_{1}}(\mathcal{K}, \Gamma)$.

We need a preparatory result. Let $\mathcal{H}$ be an infinite-dimensional complex Hilbert space. We prove that the subsets of $\mathfrak{B}(\mathcal{H})$, consisting of isometries with fixed index, are connected.

Lemma 5.1 The sets $\mathcal{I}^{n}(\mathcal{H}):=\left\{V \in \mathfrak{B}(\mathcal{H}) \mid V^{*} V=\mathbf{1}\right.$, ind $\left.V^{*}=n\right\}$ are arcwise connected in the norm topology.

Proof. Let $V, V^{\prime} \in \mathcal{I}^{n}(\mathcal{H})$. Since $\operatorname{dim} \operatorname{ker} V^{*}=\operatorname{dim} \operatorname{ker} V^{\prime *}$, there exists a unitary operator $U$ on $\mathcal{H}$ with $V^{\prime}=U V$ (choose a partial isometry $u$ with initial space $\operatorname{ker} V^{*}$ and final space $\operatorname{ker} V^{\prime *}$ and set $\left.U:=V^{\prime} V^{*}+u\right)$. Since the unitary group $\mathcal{U}(\mathcal{H})$ is arcwise connected, there exists a continuous curve $U(t)$ in $\mathcal{U}(\mathcal{H})$ with $U(0)=\mathbf{1}$ and $U(1)=U$. Then $U^{\prime}(t):=U(t) V$ is a continuous curve in $\mathcal{I}^{n}(\mathcal{H})$ with $U^{\prime}(0)=V$ and $U^{\prime}(1)=V^{\prime}$.

Let us return to $\mathcal{I}_{P_{1}}(\mathcal{K}, \Gamma)$. In the following, the shorthand $V \sim V^{\prime}$ stands for the existence of a continuous curve in $\mathcal{I}_{P_{1}}(\mathcal{K}, \Gamma)$ which connects $V$ to $V^{\prime}$. Note that " $\sim$ " is an equivalence relation and that $V \sim V^{\prime}$ implies $V V^{\prime \prime} \sim V^{\prime} V^{\prime \prime}$ and $V^{\prime \prime} V \sim V^{\prime \prime} V^{\prime}$ for $V, V^{\prime}, V^{\prime \prime} \in \mathcal{I}_{P_{1}}(\mathcal{K}, \Gamma)$.

Theorem 5.2 The connected components of $\mathcal{I}_{P_{1}}(\mathcal{K}, \Gamma)$ are precisely the sets $\mathcal{I}_{P_{1}}^{0}(\mathcal{K}, \Gamma)^{ \pm}$and $\mathcal{I}_{P_{1}}^{2 m}(\mathcal{K}, \Gamma), 1 \leq m \leq \infty$.

Proof. Let $V \in \mathcal{I}_{P_{1}}^{2 m}(\mathcal{K}, \Gamma)$, and let $V=U W$ be a decomposition as in Proposition 4.10. Then $U \in$ $\mathcal{I}_{P_{1}}^{0}(\mathcal{K}, \Gamma)^{+}$since ker $U_{11}=\{0\}$. This implies $U \sim \mathbf{1}$ by Araki's result, hence $V=U W \sim W$.

Since $V_{11}$ and $V_{11}{ }^{*}$ both have infinite rank and since $\operatorname{dim} \operatorname{ker} V_{11}=\operatorname{dim}\left(V_{12}\left(\operatorname{ker} V_{22}\right)\right)($ cf. (23) , (24)), there exists an isometry $\hat{W}_{11}$ on $\mathcal{K}_{1}$ with ind $\hat{W}_{11}{ }^{*}=\operatorname{dim}\left(\operatorname{ker} V_{11}{ }^{*} \ominus V_{12}\left(\operatorname{ker} V_{22}\right)\right)=m(\operatorname{cf}$. (25)) and

$$
\hat{W}_{11}\left(\operatorname{ran} V_{11}{ }^{*}\right)=\operatorname{ran} V_{11}, \quad \hat{W}_{11}\left(\operatorname{ker} V_{11}\right)=V_{12}\left(\operatorname{ker} V_{22}\right) .
$$

Let $\hat{W}:=\hat{W}_{11}+\Gamma \hat{W}_{11} \Gamma \in \mathcal{I}_{P_{1}}^{2 m}(\mathcal{K}, \Gamma)$ be the associated Bogoliubov operator with $\hat{W} \hat{W}^{*}=W W^{*}$. Inserting the definitions, we find that

$$
\hat{U}:=\hat{W}^{*} W \in \mathcal{I}_{P_{1}}^{0}(\mathcal{K}, \Gamma)
$$

is a unitary Bogoliubov operator with $\hat{W} \hat{U}=W$ and $\operatorname{ker} \hat{U}_{11}=\operatorname{ker} V_{11}$, hence $\chi(\hat{U})=\chi(V)$.

Now let $V^{\prime} \in \mathcal{I}_{P_{1}}^{2 m}(\mathcal{K}, \Gamma)$ be another Bogoliubov operator with corresponding operators $W^{\prime}, \hat{W}^{\prime} \in$ $\mathcal{I}_{P_{1}}^{2 m}(\mathcal{K}, \Gamma), \hat{U}^{\prime} \in \mathcal{I}_{P_{1}}^{0}(\mathcal{K}, \Gamma)$. By Lemma 5.1. $\hat{W} \sim \hat{W}^{\prime}$ since both are diagonal. Assume that $\chi(V)=\chi\left(V^{\prime}\right)$. Then $\hat{U} \sim \hat{U}^{\prime}$ by Araki's result, and we conclude

$$
V \sim W=\hat{W} \hat{U} \sim \hat{W} \hat{U}^{\prime} \sim \hat{W}^{\prime} \hat{U}^{\prime}=W^{\prime} \sim V^{\prime} .
$$

Therefore either of the two subsets $\mathcal{I}_{P_{1}}^{2 m}(\mathcal{K}, \Gamma)^{ \pm}:=\left\{V \in \mathcal{I}_{P_{1}}^{2 m}(\mathcal{K}, \Gamma) \mid \chi(V)= \pm 1\right\}$ is arcwise connected. Below, we give an example of a continuous curve in $\mathcal{I}_{P_{1}}^{2 m}(\mathcal{K}, \Gamma)$ which connects $\mathcal{I}_{P_{1}}^{2 m}(\mathcal{K}, \Gamma)^{+}$to $\mathcal{I}_{P_{1}}^{2 m}(\mathcal{K}, \Gamma)^{-}$. Hence $\mathcal{I}_{P_{1}}^{2 m}(\mathcal{K}, \Gamma)$ itself is connected. Of course, $V \sim V^{\prime}$ cannot hold if ind $V \neq$ ind $V^{\prime}$.

Example. Let $V(\varphi)$ be the Bogoliubov operator introduced in the example in Section 3.2 (with $P=P_{1}$ ). Then $V(\varphi) \in \mathcal{I}_{P_{1}}^{2}(\mathcal{K}, \Gamma)$ since $V(\varphi)_{12}{ }^{*} V(\varphi)_{12}=\left(S_{V(\varphi)}\right)_{22}=\left(1-\lambda_{\varphi}\right) \overline{E_{0}}$ has finite rank, and $\varphi \mapsto V(\varphi)$ is a continuous curve in $\mathcal{I}_{P_{1}}^{2}(\mathcal{K}, \Gamma)$. We have $\operatorname{ker} V(\varphi)_{11}=\operatorname{ker}\left(S_{V(\varphi)}\right)_{11}=\operatorname{ker}\left(\lambda_{\varphi} E_{0}+\sum_{n \geq 1} E_{n}\right)$, hence

$$
\chi(V(\varphi))=\left\{\begin{aligned}
1, & \varphi \notin(4 \mathbb{Z}+3) \pi / 4 \\
-1, & \varphi \in(4 \mathbb{Z}+3) \pi / 4
\end{aligned}\right.
$$


Let $V \in \mathcal{I}_{P_{1}}^{2 m-2}(\mathcal{K}, \Gamma)$ with $\left[P_{1}, V\right]=0$. Then $\chi(V V(\varphi))=\chi(V(\varphi))$ since $V_{11}$ is isometric, so $\varphi \mapsto V V(\varphi)$ is a continuous curve in $\mathcal{I}_{P_{1}}^{2 m}(\mathcal{K}, \Gamma)$ which connects $\mathcal{I}_{P_{1}}^{2 m}(\mathcal{K}, \Gamma)^{+}$to $\mathcal{I}_{P_{1}}^{2 m}(\mathcal{K}, \Gamma)^{-}$. This completes the proof of Theorem 5.2 .

$V(\varphi)$ may also serve to illustrate that $\chi$ is not multiplicative on $\mathcal{I}_{P_{1}}(\mathcal{K}, \Gamma)$. Define a Bogoliubov operator $U:=2^{-1 / 2} f_{0}^{+}\left\langle f_{0}^{+}+f_{1}^{-},.\right\rangle-2^{-1 / 2} f_{1}^{+}\left\langle f_{0}^{-}-f_{1}^{+},.\right\rangle+2^{-1 / 2} f_{0}^{-}\left\langle f_{0}^{-}+f_{1}^{+},.\right\rangle-2^{-1 / 2} f_{1}^{-}\left\langle f_{0}^{+}-f_{1}^{-},.\right\rangle+$ $\sum_{n \geq 2}\left(E_{n}+\overline{E_{n}}\right)$. Then $U \in \mathcal{I}_{P_{1}}^{0}(\mathcal{K}, \Gamma)$, and a calculation shows that $U_{11}=\frac{1}{\sqrt{2}}\left(E_{0}+E_{1}\right)+\sum_{n \geq 2} E_{n}$ and $U V\left(\frac{3 \pi}{4}\right)=V\left(\frac{\pi}{2}\right)$. This entails

$$
1=\chi\left(U V\left(\frac{3 \pi}{4}\right)\right) \neq \chi(U) \chi\left(V\left(\frac{3 \pi}{4}\right)\right)=-1
$$

since $\operatorname{ker} U_{11}=\operatorname{ker} V\left(\frac{\pi}{2}\right)_{11}=\{0\}$, but $\operatorname{ker} V\left(\frac{3 \pi}{4}\right)_{11}=\mathbb{C} f_{0}$. We finally note that the eigenvalues $\pm\left(1-\lambda_{\varphi}\right)$ of $P_{1}-S_{V(\varphi)}=\left(1-\lambda_{\varphi}\right)\left(E_{0}-\overline{E_{0}}\right)$ have multiplicity one if $\lambda_{\varphi} \neq 1$, in contrast to the unitary case where the multiplicities of eigenvalues in $(0,1)$ are always even [15].

Acknowledgments. I would like to thank Professor K. Fredenhagen for the initiation of this work and for many helpful discussions. Thanks for discussions are also due to J. Böckenhauer and to my colleagues at the FU Berlin.

\section{References}

[1] D. Shale, W. F. Stinespring, "Spinor representations of infinite orthogonal groups", J. Math. Mech. 14,1, 315 (1965).

[2] K. O. Friedrichs, Mathematical Aspects of the Quantum Theory of Fields, Interscience Publ., New York, 1953.

[3] F.A. Berezin, The Method of Second Quantization, Academic Press, New York, 1966.

[4] G. Labonté, "On the nature of 'strong' Bogoliubov transformations for fermions", Commun. Math. Phys. 36, 59 (1974).

[5] K. Fredenhagen, "Implementation of automorphisms and derivations of the CAR-algebra", Commun. Math. Phys. 52, 255 (1977).

[6] M. Klaus, G. Scharf, "The regular external field problem in quantum electrodynamics", Helv. Phys. Acta 50, 779 (1977).

[7] S. N. M. Ruijsenaars, "On Bogoliubov transformations for systems of relativistic charged particles", J. Math. Phys. 18, 517 (1977).

[8] S. N. M. Ruijsenaars, "On Bogoliubov transformations. II. The general case", Ann. Phys. 116, 105 (1978).

[9] S. Doplicher, J. E. Roberts, "Fields, statistics and non-abelian gauge groups", Commun. Math. Phys. 28, 331 (1972); "Why there is a field algebra with a compact gauge group describing the superselection structure in particle physics", Commun. Math. Phys. 131, 51 (1990);

J. E. Roberts, "Cross products of von Neumann algebras by group duals", Sympos. Math. 20, 335 (1976).

[10] R. Haag, Local Quantum Physics, Springer-Verlag, Berlin, Heidelberg, New York, 1992. 
[11] R. T. Powers, E. Størmer, "Free states of the canonical anticommutation relations", Commun. Math. Phys. 16, 1 (1970).

[12] H. Araki, "On quasifree states of CAR and Bogoliubov automorphisms", Publ. RIMS Kyoto Univ. 6, $385(1970 / 71)$.

[13] Y. Watatani, "Index for C*-subalgebras", Memoirs of the Am. Math. Soc. Vol. 83 No. 424 (1990).

[14] R. Longo, "Index of subfactors and statistics of quantum fields", I: Commun. Math. Phys. 126, 217 (1989); II: Commun. Math. Phys. 130, 285 (1990).

[15] H. Araki, "Bogoliubov automorphisms and Fock representations of canonical anticommutation relations", in Operator Algebras and Mathematical Physics, Am. Math. Soc. Vol. 62, 23 (1987).

[16] C. Binnenhei, "Bogoliubov-Transformationen und lokalisierte Morphismen für freie Diracfelder", BONN-IR-93-33.

[17] G. Mack, V. Schomerus, "Conformal field algebras with quantum symmetry from the theory of superselection sectors", Commun. Math. Phys. 134, 139 (1990).

[18] J. Fuchs, A. Ganchev, P. Vecsernyés, "Level 1 WZW superselection sectors", Commun. Math. Phys. 146, 553 (1992).

[19] J. M. Böckenhauer, "Localized endomorphisms of the chiral Ising model", DESY 94-116.

[20] H. Araki, "On the diagonalization of a bilinear Hamiltonian by a Bogoliubov transformation", Publ. RIMS Kyoto Univ. 4, 387 (1968).

[21] D. Shale, W. F. Stinespring, "States of the Clifford algebra", Ann. Math. 80, 365 (1964).

[22] J. M. Böckenhauer, "Decomposition of representations of CAR induced by Bogoliubov endomorphisms", DESY 94-173.

[23] A. L. Carey, C. A. Hurst, D. M. O'Brien, "Automorphisms of the canonical anticommutation relations and index theory", J. Funct. Anal. 48, 360 (1982).

[24] J. Manuceau, "Étude algébrique des états quasi-libres", in Cargèse Lectures in Physics Vol. 4 (ed. Kastler), Gordon and Breach, New York, London, Paris, 1970.

[25] T. Kato, Perturbation Theory for Linear Operators, Springer-Verlag, Berlin, Heidelberg, New York, 1966.

[26] J. Manuceau, F. Rocca, D. Testard, "On the product form of quasi-free states", Commun. Math. Phys. 12, 43 (1969).

[27] O. Bratteli, D. W. Robinson, Operator Algebras and Quantum Statistical Mechanics. Vol. 2: Equilibrium States, Models in Quantum Statistical Mechanics, Springer-Verlag, Berlin, Heidelberg, New York, 1981.

[28] D. Husemoller, Fibre Bundles, $2^{\text {nd }}$ ed.: Springer-Verlag, New York, Heidelberg, Berlin, 1975.

[29] R. T. Powers, "A non spatial continuous semigroup of *-endomorphisms of $\mathfrak{B}(\mathcal{H})$ ", Publ. RIMS Kyoto Univ. 23, 1053 (1987). 
[30] J. Cuntz, "Simple C*-algebras generated by isometries", Commun. Math. Phys. 57, 173 (1977).

[31] K. Fredenhagen, "Observables, superselection sectors and gauge groups", in New Symmetry Principles in Quantum Field Theory, Proc. of Cargèse Summer Inst., 1991.

[32] G. Rideau, "On some representations of the anticommutation relations", Commun. Math. Phys. 9, 229 (1968).

[33] R. T. Powers, "Representations of the canonical anticommutation relations", Thesis Princeton Univ. 1967

[34] A. L. Carey, S. N. M. Ruijsenaars, "On fermion gauge groups, current algebras and Kac-Moody algebras", Acta Appl. Math. 10, 1 (1987). 